\title{
Collective coordinate descriptions of magnetic domain wall motion in perpendicularly magnetized nanostructures under the application of in-plane fields
}

\author{
S. Ali Nasseri ${ }^{\mathrm{a}, \mathrm{b}, *}$, Eduardo Martinez ${ }^{\mathrm{c}}$, Gianfranco Durin ${ }^{\mathrm{a}, \mathrm{d}}$ \\ a ISI Foundation, Via Chisola 5, 10126 Torino, Italy \\ b Politecnico di Torino, Corso Duca degli Abruzzi 24, 10129 Torino, Italy \\ ${ }^{\mathrm{c}}$ University of Salamanca, Dpto Fisica Aplicada, Plaza de los Caidos s/n., E37008 Salamanca, Spain \\ d Istituto Nazionale di Ricerca Metrologica (INRiM), Strada delle Cacce 91, 10135 Torino, Italy
}

\section{A R T I C L E I N F O}

\section{Keywords:}

Domain wall motion

Spintronics

Collective coordinate modeling

\begin{abstract}
A B S T R A C T
Manipulation of magnetic domain walls in nanostructures can be used to improve the capabilities of the next generation of memory and sensing devices. Materials of interest for such devices include heterostructures of ultrathin ferromagnets sandwiched between a heavy metal and an oxide, where spin-orbit coupling and broken inversion symmetry give rise to the Dzyaloshinskii-Moriya interaction (DMI), stabilizing chiral domain walls. The efficiency of the motion of these chiral domain walls may be controlled using in-plane magnetic fields. This property has been used both for measurement of DMI strength, and for improved performance in applications. While micromagnetic simulations are able to accurately predict domain wall motion under in-plane fields in these materials, collective coordinate models such as the $q-\phi$ and $q-\phi-\chi$ models fail to reproduce the micromagnetic results. In this theoretical work, we present a set of extended collective coordinate models including canting in the domains, which better reproduce micromagnetic results, and improve our understanding of the effect of in-plane fields on magnetic domain walls. These models are used in conjunction with micromagnetic simulations to develop simpler descriptions of DW motion under specific conditions. Our new models and results help in the development of future domain wall based devices based on perpendicularly magnetized materials.
\end{abstract}

\section{Introduction}

Manipulating magnetic domain walls (DWs) within nanostructures has been linked with the development of spintronic logic [1,2], memory [3-6] and sensing [7] devices. The next generation of magnetic memory and storage devices could rely on DWs moving along magnetic tracks or wires, with different principles for such devices being explored to achieve mass storage without the need for mechanical moving parts $[4,5]$. Simulation capabilities are key to better understand the underlying processes in these systems, and to assess different design concepts. The main computational framework to analyze these ferromagnetic systems is based on the Landau-Lifshitz-Gilbert (LLG) equation which is applicable to a wide range of problems in magnetism, including DW motion. However, the use of micromagnetic simulations specially for large samples is computationally costly and time consuming, as the numerical solution for the magnetic configuration needs to be determined both spatially and temporally.

Alternatively, simpler models may be extracted from the LLG equation to analyze the motion of specific topological defects of interest, such as vortices and DWs [8-17]. The simplified nature of these collective coordinate models (CCMs) is due to the introduction of an ansatz which characterizes the structure of the spin texture of interest. In 1972, Slonczewski used a Lagrangian approach to propose a CCM for analyzing DW motion in perpendicularly magnetized materials (the $q-\phi$ model) [8]. This model relates the DW position ( $q$ ), and the DW's supposedly uniform magnetization $(\phi)$ to the different interactions affecting the system. Thiaville and Nakatani later extended the $q-\phi$ model to in-plane systems and introduced the DW width parameter $(\Delta)$ as an additional time varying coordinate, leading to the $q-\phi-\Delta$ model [13]. However, their findings showed that the evolution of $\Delta$ has minimal effect on the dynamics and could be neglected. Due to interest in current-driven DW motion at the time, the spin-transfer torque (STT) mechanism was also implemented as part of these newer models $[11,12]$.

Recent studies on DW motion have focused on perpendicular magnetic anisotropy (PMA) heterostructures in which ultrathin

\footnotetext{
* Corresponding author at: ISI Foundation, Via Chisola 5, 10126 Torino, Italy.

E-mail address: ali.nasseri@isi.it (S.A. Nasseri).
} 
ferromagnets are sandwiched between a heavy metal layer and an oxide $(H M / F M / O x)$. In these structures, spin-orbit coupling (SOC) and broken inversion symmetry (BIC) modify the static structure of the DW and contribute to DW motion [18-22]. Specifically, the DzyaloshinskiMoriya interaction (DMI) present in these systems stabilizes Néel DW structures of specific chirality. SOC has also been linked to enhanced current induced DW motion, with the spin Hall effect (SHE) suggested as the dominant mechanism for this observation [22]. Moving DWs tend to tilt in the plane of the sample in these systems, with the $q-\phi-\chi$ model (where $\chi$ denotes the DW tilting) developed to describe DW motion in these systems [14].

The efficiency of DW motion depends on the internal magnetic structure of the DW. As such, applied fields in-plane of the sample can be used to control DW chirality, enhancing the efficiency of currentdriven DW motion [14,17,23-29]. While micromagnetic simulations of this problem are in agreement with experiments, conventional CCMs (such as the $q-\phi$ and $q-\phi-\chi$ models) fail to reproduce the micromagnetic results $[17,24]$.

Despite this shortcoming, equations derived from the $q-\phi-\chi$ model are used in two of the most prominent methods of assessing the strength of the DMI, both of which rely on the manipulation of DW dynamics under in-plane magnetic fields. In the first and most common method, magnetic bubbles are expanded in the thin film of interest under the application of in-plane and out-of-plane fields in the creep regime $[30,31]$. This method assumes that the points with significant Néel character are located on the axis of the applied in-plane field, and the DMI field is assumed to be equal to the in-plane field which reverses the chirality of the DW. A second method of assessing DMI strength uses a critical longitudinal field which can be identified in current-driven DW motion in nanowires with DMI; at the critical point the DW is locked in place irrespective of applied current, and the value of the longitudinal field at that point is related to the DMI strength [17]. While most experimentalists rely on the $q-\phi-\chi$ model in DMI strength measurements using the methods above, as mentioned previously, these models seem to not be accurate as they cannot reproduce the micromagnetic results. This calls for improvements in collective coordinate modeling of DW motion, both to reproduce micromagnetic results and to help in the assessment of DMI strength in material stacks.

In our previous work [17], we developed an extended collective coordinate model which better reproduced micromagnetic results in the case of current-driven DW motion in PMA systems with strong DMI under the application of in-plane fields. This model was developed based on the Bloch profile and had four collective coordinates $(q, \phi, \chi, \Delta)$. The increased accuracy of the model was attributed to the inclusion of an approximation of canting in the domains as an additional parameter in the CCM. Canting in the domains arises from the application of in-plane fields to the system, and was included in the limits of integration when deriving the CCM.

In this paper, we present a new extended set of CCMs based on an inherently canted ansatz to describe DW motion in PMA systems with DMI. We compare this model in mathematical form to past models present in the literature $[8,13,14,17]$. The models presented in this work are used to study two material stacks, which differ in the strength of DMI and uniaxial anisotropy. Specifically, we find that while our past studies showed that only a four coordinate model can correctly predict the characteristic shape of the DW velocity curve [17], our new canted models maintains higher accuracy when only two coordinates, namely the DW position $(q)$ and magnetization angle $(\phi)$ are used. This highlights the rigidity of the DW during motion, and the fact that canting in the domains plays an important role in magnetic DW motion under inplane fields. We also found that the anisotropy of the system plays an important role in the applicability of the models, with minimal difference observed between the different models in systems with high anisotropy (which corresponds to low canting and narrower DWs).

We also showcase in detail the impact of in-plane fields on field- and current-driven DW motion, identifying critical in-plane fields which
Table 1

Material parameters of the two systems studied in this work. The DMI strength of the $\mathrm{Pt} / \mathrm{CoFe} / \mathrm{MgO}$ sample is twice that of the $\mathrm{Pt} / \mathrm{Co} / \mathrm{Ni} / \mathrm{Co} / \mathrm{MgO} / \mathrm{Pt}$ sample, while its PMA constant is $1 / 3$ that of the later sample. This difference in material properties helps better understand their effects on DW dynamics.

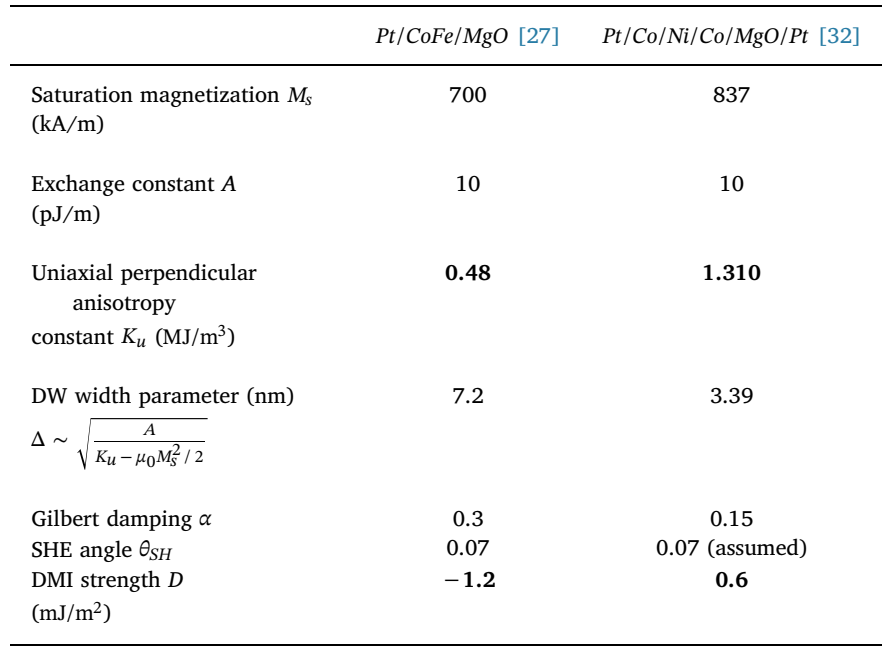

lead to effects such as no tilting, no movement or a Bloch DW structure in DW dynamics. Analytical solutions are proposed based on the CCMs for these critical points that shed some light on the physics involved, and show how these points could help in measuring the strength of various interactions in experiments.

\section{Methods}

\subsection{Systems under study}

In this work, we study two $2.8 \mu \mathrm{m}$ long, $160 \mathrm{~m}$ wide nanowires with the magnetic properties listed in Table 1 and a $0.6 \mathrm{~nm}$ thickness for the ferromagnetic layer. These samples were selected as they both have DMI and PMA; however, the DMI strength of the $\mathrm{Pt} / \mathrm{CoFe} / \mathrm{MgO}$ sample is twice that of the $\mathrm{Pt} / \mathrm{Co} / \mathrm{Ni} / \mathrm{Co} / \mathrm{MgO} / \mathrm{Pt}$ sample, while its PMA constant is $1 / 3$ that of the later sample. This helps us better understand the impact of these two parameters on the structure and dynamics of DWs.

\subsection{Micromagnetic simulations}

To understand the magnetization dynamics in these samples, we conducted micromagnetic simulations using Mumax $^{3}$ [33] which numerically solves the Landau-Lifshitz-Gilbert (LLG) equation. A micromagnetic cell size of $1 \mathrm{~nm} \times 1 \mathrm{~nm} \times 0.6 \mathrm{~nm}$ was used for all micromagnetic simulations.

As we are interested in magnetic DW dynamics under applied fields and currents in a perpendicularly magnetized heterostructure, the DMI [34-36], spin-orbit torques (SOTs) [37-39], and the spin transfer torque (STT) mechanism $[12,40]$ were included in addition to the traditional interactions included in the effective field (exchange, anisotropy, magnetostatics, and the Zeeman energy). With these terms, the LLG will take the following form:

$$
\begin{aligned}
\frac{d \vec{m}}{d t} & =-\gamma \vec{m} \times \vec{H}_{\text {eff }} \overbrace{+\alpha \vec{m} \times \frac{d \vec{m}}{d t}}^{\text {Damping term }} \\
& \overbrace{-(\vec{u} \cdot \nabla) \vec{m}}^{\text {adiabatic STT }}+\overbrace{\beta \vec{m} \times\left(\left(\begin{array}{c}
\vec{u} \cdot \nabla) \vec{m}) \\
\text { non-adiabatic STT }
\end{array}\right.\right.}^{\text {Slonczewski-like SOT }} \\
& +\overbrace{\gamma H_{F L}\left(\vec{m} \times \hat{u}_{S O T}\right)}^{\text {field-like SOT }}-\overbrace{\gamma H_{S L} \vec{m} \times\left(\vec{m} \times \hat{u}_{S O T}\right)}^{\text {s. }}
\end{aligned}
$$

with $H_{F L}$ denoting the field-like components of the spin-orbit torques, $H_{S L}$ denoting the Slonczewski-like component of the spin-orbit torques, 
$\hat{u}_{\text {SOT }}=\widehat{J} \times \hat{n}$ is the direction of spin current where $\widehat{J}$ is the direction of current flow in the heavy metal layer and $\hat{n}$ is the interface normal. In this equation $\beta$ is the nonadiabaticity coefficient and $\vec{u}=\frac{\vec{J} P g \mu_{b}}{2 e M_{s}}$ denotes the velocity of the electrons under a current density of $J$ and polarization rate of $P$. The effective field is linked with the internal energy of the system through

$\vec{H}_{e f f}=\frac{1}{\mu_{0} M_{s}} \frac{\delta \Omega}{\delta \vec{m}}$

The internal energy density of the magnetic system $(\Omega)$ can be calculated using:

$$
\begin{aligned}
\Omega= & \overbrace{A \sum_{i=1}^{3}\left|\nabla m_{i}\right|^{2}}^{\text {Exchange }}+\overbrace{K_{U} \sin ^{2} \theta}^{\text {Anisotropy }}-\frac{\overbrace{\frac{\mu_{0} M_{s}}{2} \overrightarrow{H_{d}} \cdot \vec{m}}^{\text {Magnetostatics }}}{-\overbrace{\mu_{0} M_{s} \overrightarrow{H_{a}} \cdot \vec{m}}^{\text {Zeeman }}} \\
& +\overbrace{D\left(m_{z} \nabla \cdot \vec{m}-(\vec{m} \cdot \nabla) m_{z}\right)}^{D M I}
\end{aligned}
$$

While the LLG equation can grasp the intricate details of the dynamics, the fact that magnetization has to be determined spatially and temporally at every point within the system translates to long computation times, limiting the usefulness of this description for fast calculations.

\subsection{Collective coordinate modeling}

To better understand the underlying physics observed in the motion of DWs under in-plane fields in these materials, we developed extended collective coordinate models (CCMs) using the Euler-Lagrange equation based on the Lagrangian and dissipation functions presented in the literature $[14,17]$.

\subsubsection{Collective coordinates}

Based on micromagnetic simulations, experimental observations and previous work $[8,13,14]$, we selected four time dependent collective coordinates to describe the collective behavior of the DW:

1. The position of the center of the DW $(q)$;

2. Magnetization angle at the center of the DW (assumed to be homogeneous) $(\phi)$;

3. The DW width parameter $(\Delta)$;

4. The geometric tilt angle of the DW $(\chi)$.

Using these collective coordinates, the DW is modeled as a thin line with four degrees of freedom, as defined in Fig. 1a. As long as the DW maintains its shape, this description is valid.

\subsubsection{Characterizing the DW structure}

The collective coordinates need to be linked with spherical coordinates of the magnetization (Fig. 1b) in order to write the Lagrangian and dissipation functions in terms of these coordinates. Based on energy minimization in the static system, the following two profiles may be derived to connect the two coordinate systems and introduce the DW in our models:

1. Ansatz 1 (tilted Bloch profile [14]): $\tan \frac{\theta}{2}=\exp (Z)$

2. Ansatz 2 (inherently canted profile, a continuous version of an ansatz previously used in the literature [41-44]):
$\tan \left(\frac{\theta+\theta_{c}}{2}\right)=\frac{\exp (Z)+\sin \theta_{c}}{\cos \theta_{c}}$.

with $Z=\frac{(x-q) \cos \chi+y \sin \chi}{p_{w} \Delta}$.The parameter $p_{w}= \pm 1$ is used to adjust for up-down vs down-up DWs. In both cases, we also assume that magnetization is constant along the DW, hence $\psi(r, t)=\phi(t)$.

The difference between the two ansatzes is that ansatz 2 takes into account the canting in the domains due to the application of in-plane fields in the profile itself, a feature which was observed to play an important role in DW motion under in-plane fields [17]. In the presence of an in-plane field, the magnetization in the domains may be described in spherical coordinates as $\psi=\operatorname{atan}\left(\frac{H_{y}}{H_{x}}\right)$ and $\sin \theta_{c}=\operatorname{Sgn}\left(H_{i} p\right) \frac{\mu_{0} M_{S}\left(H_{x} \cos \phi+H_{y} \sin \phi\right)}{2 K_{u}+\mu_{0} M_{S}^{2}\left(N_{x, d} \cos ^{2} \phi+N_{y, d} \sin ^{2} \phi-N_{z, d}\right)}$ where $\theta_{c}$ is the value of the canting angle, $H_{i} p$ is the in-plane field and $N_{x, d}, N_{y, d}, N_{z, d}$ denote the demagnetizing factors felt by the spins in the domain [17]. This description may be derived through energy minimization in the domains. Note that, as we are projecting the 3-D magnetization angle onto a plane, a sign for the canting angle $\theta_{c}$ also needs to be included in the model (in the form of $\operatorname{Sgn}\left(H_{i} p\right)$ ); we defined the angle to be negative for negative in-plane fields and positive for positive in-plane fields. Comparison to cases where this sign was not taken into account later revealed the importance of this convention to improve model accuracy and continuity. We believe this convention is needed, as the DW will be wider when fields are applied opposite to the direction of chirality of the DW.

Fig. 2 shows the accuracy of these ansatzes in predicting the static structure of the DWs compared to micromagnetic simulations $(\mu \mathrm{m})$. We know from past studies that the $\theta$ component of magnetization does not change significantly under dynamic conditions; as such the DW can be assumed to maintain the same structure during motion. According to Fig. 2, under the same in-plane field the domains in the $\mathrm{Pt} / \mathrm{Co} / \mathrm{Ni} / \mathrm{Co} / \mathrm{MgO} / \mathrm{Pt}$ show lower canting (about $10^{\circ}$ in this case), which can be attributed to the higher uniaxial anisotropy of this system. We can deduce from this observation that canting will have a much lower impact on DW motion in this material, and the models including canting will not differ dramatically from those without canting. In contrast, in the $\mathrm{Pt} / \mathrm{CoFe} / \mathrm{MgO}$ sample (Fig. 2a) the lower anisotropy leads to high canting in the domains (about $40^{\circ}$ in this case). In addition, similar simulations showed that the DW width parameter changes more dramatically in the $\mathrm{Pt} / \mathrm{CoFe} / \mathrm{MgO}$ compared to $\mathrm{Pt} / \mathrm{Co} / \mathrm{Ni} / \mathrm{Co} / \mathrm{MgO} / \mathrm{Pt}$ due to the differences in the uniaxial anisotropy of the two systems.

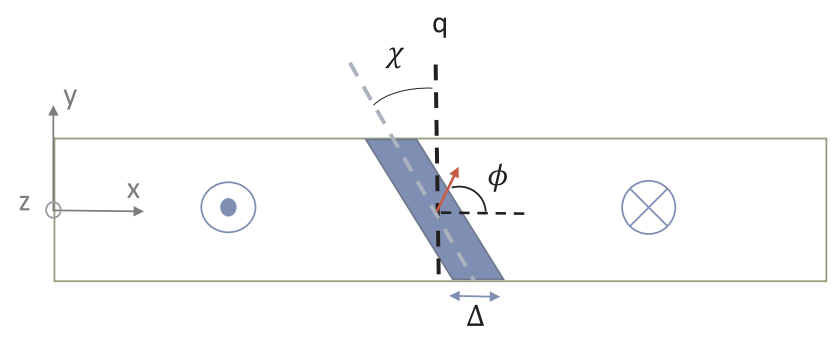

(a) Collective coordinates.

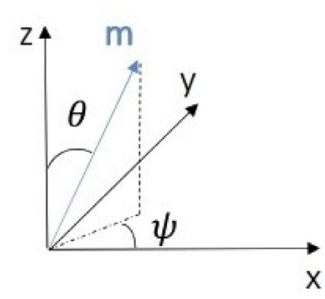

(b) Spherical coordinates.

Fig. 1. Coordinate systems used in this work. (a) The collective coordinates used to describe the DW. (b) The spherical coordinates describing the magnetization at every point in the system. 


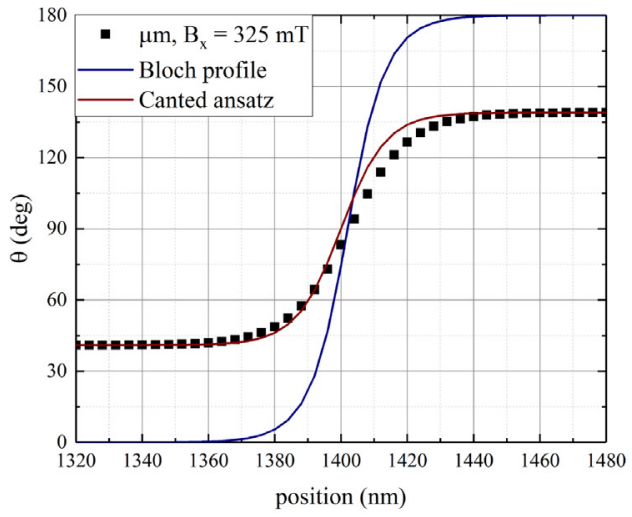

(a) DW profile of $\mathrm{Pt} / \mathrm{CoFe} / \mathrm{MgO}$.

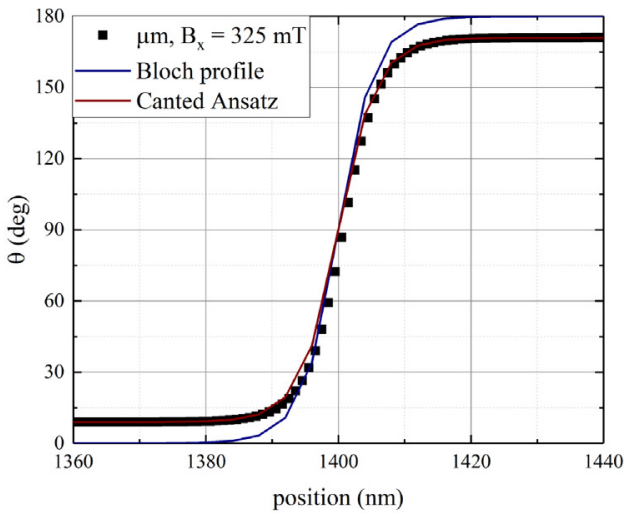

(b) DW profile of $\mathrm{Pt} / \mathrm{Ni} / \mathrm{Co} / \mathrm{Ni} / \mathrm{MgO} / \mathrm{Pt}$.

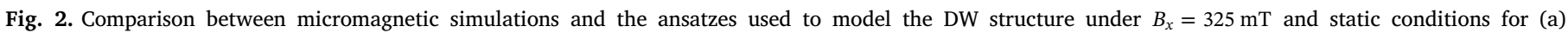
$\mathrm{Pt} / \mathrm{CoFe} / \mathrm{MgO}$, and (b) $\mathrm{Pt} / \mathrm{Co} / \mathrm{Ni} / \mathrm{Co} / \mathrm{MgO} / \mathrm{Pt}$. The magnetization angle $\theta=\operatorname{acos}\left(m_{z}\right)$ was calculated in the middle of the wire.

Overall, these simulations show that systems with very high anisotropies are less affected by in-plane fields, and effects such as canting and DW width change due to the application of in-plane fields will have a smaller impact on the structure of the DWs in these systems.

\subsubsection{Dynamic modeling}

In order to evaluate DW dynamics using the collective coordinates, we need to rewrite the energy terms using these coordinates and the properties of the ansatz. The energy and dissipation function then need to be integrated along the length and width of the wire, and plugged into the Euler-Lagrange equation. This process will result in four equations:

$$
\begin{aligned}
\alpha I_{1} \frac{\dot{q}}{p_{w} \Delta} \cos \chi+I_{2} \dot{\phi}= & \mu_{0} \gamma\left(I_{2} H_{z}-I_{3} H_{S L}\left[\sin \phi u_{S O T, x}-\cos \phi u_{S O T, y}\right]\right) \\
& +\beta I_{1} \frac{u}{p_{w} \Delta} \cos \chi
\end{aligned}
$$

$I_{2} \frac{\dot{q}}{p_{w} \Delta} \cos \chi-\alpha I_{4} \dot{\phi}=I_{4} \frac{\mu_{0} \gamma M_{s}}{2}\left(N_{y}-N_{x}\right) \sin 2(\phi-\chi)+I_{5} \frac{u}{p_{w} \Delta} \cos \chi$

$$
+I_{6} \mu_{0} \gamma\left[H_{x} \sin \phi-H_{y} \cos \phi\right]-I_{3} \frac{\gamma D}{M_{s} p_{w} \Delta} \sin (\phi-\chi)
$$

$\alpha I_{7}\left[\frac{\dot{\Delta}}{p_{w} \Delta}+\dot{\chi} \tan \chi\right]=\frac{\gamma}{M_{s}}\left[I_{1} \frac{A}{\left(p_{w} \Delta\right)^{2}}-I_{4} p_{w} K\right]$

$$
+I_{6} \mu_{0} \gamma p_{w}\left(H_{x} \cos \phi+H_{y} \sin \phi\right)
$$

$$
\begin{aligned}
-\alpha I_{7} & {\left[\frac{\dot{\Delta}}{p_{w} \Delta} \sin \chi+\frac{\dot{\chi}}{\cos \chi}\left[\frac{I_{1}}{6 I_{7}}\left(\frac{w}{p_{w} \Delta}\right)^{2}+\sin ^{2} \chi\right]\right] } \\
= & \frac{\gamma}{M_{s}} \sin \chi\left[I_{1} \frac{A}{\left(p_{w} \Delta\right)^{2}}+I_{4} K-I_{6} \mu_{0} M_{s}\left(H_{x} \cos \phi+H_{y} \sin \phi\right)\right] \\
& +I_{4} \frac{\mu_{0} \gamma M_{s}}{2} \cos \chi\left(N_{x}-N_{y}\right) \sin 2(\phi-\chi)+I_{3} \frac{\gamma D}{M_{s} p_{w} \Delta} \sin \phi
\end{aligned}
$$

where $K=K_{u}+\frac{\mu_{0} M_{s}^{2}}{2}\left[N_{x} \cos ^{2}(\phi-\chi)+N_{y} \sin ^{2}(\phi-\chi)-N_{z}\right]$ and $N_{x}, N_{y}$ and $N_{z}$ are the demagnetizing factors of the DW assumed to be of ellipsoidal form [45]. For the cases of interest in this paper, we assumed that the spin Hall effect gives rise to a Slonczewski-like field (meaning $H_{F L} \sim 0$ ). The strength of the SHE fields can be calculated using $H_{S L}=\frac{\hbar \theta_{S H E}}{2 \mu_{0} e M_{s} t_{f}}$ where $t_{f}$ is the thickness of the ferromagnetic layer [39].

Note that Eqs. (3)-(6) may be used as part of two, three or four coordinate models, meaning:

1. Assuming $\chi=0$ and a fixed $\Delta$ leads to a two coordinate model similar to the $q-\phi$ model [8] with Eqs. (3) and (4) describing the dynamics.
2. Assuming $\chi=0$ leads to a three coordinate model similar to the $q-\phi-\Delta$ model [13] with Eqs. (3)-(5) describing the dynamics.

3. Assuming a fixed $\Delta$ leads to a three coordinate model similar to the $q-\phi-\chi$ model [14] with Eqs. (3), (4), and (6) describing the dynamics.

4. Without any assumptions for the parameters, we have a four coordinate model.

As such, with each ansatz a set or class of models is really developed. For simplicity, in this paper whenever the coordinates involved are not mentioned in the model name, we are discussing a property that applied to all models in the set.

In the equations above, $I_{i} \mathrm{~s}$ are integration constants which depend on the ansatz used and relate to the amount of canting in the domains. Three classes of models can be derived:

1. Integrating ansatz 1 from 0 to $\pi$ : This model does not take into account the canting in the domains, and was presented in one of our previous works [16].

2. Integrating ansatz 1 from $\theta_{c}$ to $\pi-\theta_{c}$ : This model approximates the effect of the canting in the domains and was presented in our past work [17].

3. Integrating ansatz 2 from $\theta_{c}$ to $\pi-\theta_{c}$ : We expect this model to be the most accurate, as it takes into account the effect of canting not just in the domains but also on the DW structure.

Table 2 summarizes the value of the $I_{i}$ s for the three different groups of models. For model set 2, the closed form of the $I_{7}$ parameter is:

\section{Table 2}

Summary of model parameters derived from integration (taking into account the effects of canting and the ansatz used) for the three different model sets. Model set 1 is the model based on the Bloch profile without canting, model set 2 is also based on the Bloch profile but takes into account canting in the domains through the canting angle in the domains $\left(\theta_{c}\right)$, and model set 3 is based on an inherently canted ansatz.

\begin{tabular}{cccc}
\hline & Model 1 & Model 2 & Model 3 \\
\hline$I_{1}$ & 1 & $\cos \theta_{c}$ & $1-\left(\pi / 2-\theta_{c}\right) \tan \theta_{c}$ \\
$I_{2}$ & 1 & 1 & $\cos \theta_{c}$ \\
$I_{3}$ & $\pi / 2$ & $\pi / 2-\theta_{c}$ & $\pi / 2-\theta_{c}$ \\
$I_{4}$ & 1 & $\cos \theta_{c}$ & $\cos ^{2} \theta_{c}+\left(\pi / 2-\theta_{c}\right) \sin \theta_{c} \cos \theta_{c}$ \\
$I_{5}$ & 1 & $\cos \theta_{c}$ & $\cos \theta_{c}$ \\
$I_{6}$ & $\pi / 2$ & $\pi / 2-\theta_{c}$ & $\pi / 2-\theta_{c}$ \\
$I_{7}$ & $\pi^{2} / 6$ & Eq. (7) & Eq. (8) \\
\hline
\end{tabular}




$$
\begin{aligned}
I_{7}= & {\left[\operatorname{Li}_{2}\left(-\cos \frac{\theta_{c}}{2}\right)-\operatorname{Li}_{2}\left(-\sin \frac{\theta_{c}}{2}\right)-\operatorname{Li}_{2}\left(1-\cos \frac{\theta_{c}}{2}\right)+\operatorname{Li}_{2}\left(1-\sin \frac{\theta_{c}}{2}\right)\right] } \\
& -\cos ^{2} \frac{\theta_{c}}{2}\left[1-\log \left(\cos \frac{\theta_{c}}{2}\right)\right]+\sin ^{2} \frac{\theta_{c}}{2}\left[1-\log \left(\sin \frac{\theta_{c}}{2}\right)\right] \\
& -\cos ^{2} \frac{\theta_{c}}{2} \log \left(\sin ^{2} \frac{\theta_{c}}{2}\right)\left[\log \left(\cos \frac{\theta_{c}}{2}\right)-\frac{1}{2}\right] \\
& +\sin ^{2} \frac{\theta_{c}}{2} \log \left(\cos ^{2} \frac{\theta_{c}}{2}\right)\left[\log \left(\sin \frac{\theta_{c}}{2}\right)-\frac{1}{2}\right]-\frac{1}{2} \log \left(\sin ^{2} \frac{\theta_{c}}{2}\right) \\
& +\frac{1}{2} \log \left(\cos ^{2} \frac{\theta_{c}}{2}\right)+\log \left(\cos \frac{\theta_{c}}{2}\right) \log \left(1+\cos \frac{\theta_{c}}{2}\right) \\
& -\log \left(\sin \frac{\theta_{c}}{2}\right) \log \left(1+\sin \frac{\theta_{c}}{2}\right)+\cos \theta_{c}+\left(\cos \theta_{c}+1\right) \ln ^{2}\left(\cos \frac{\theta_{c}}{2}\right) \\
& +\left(\cos \theta_{c}-1\right) \ln ^{2}\left(\sin \frac{\theta_{c}}{2}\right)
\end{aligned}
$$

where $\mathrm{Li}_{2}$ is the polylogarithm function of order 2 .

A closed form for $I_{7}$ could not be derived for model set 3. Instead, the integral was numerically solved and fitted to the following polynomial function $\left(\mathrm{R}^{2}=1\right.$, RMSE $\left.=3.82 \times 10^{-4}\right)$

$I_{7}=0.568 \theta_{c}^{3}-0.4232 \theta_{c}^{2}-1.47 \theta_{c}+1.649$

The mathematical form of the $I_{i}$ s reveal the differences between the model sets. Fig. 3 visually depicts the differences between these parameters. While both model sets 2 and 3 reproduce the $I_{i}$ parameters for model set 1 in the limit $\theta_{c}=0$ (except for $I_{7}$ which is different depending on the ansatz), there is considerable difference between the models for the behavior of parameters $I_{1}, I_{4}, I_{5}$ and $I_{6}$ for non-zero canting angles. Not only the parameters for model sets 2 and 3 do not predict the same value for the same amount of canting, but also their behavior as a function of the canting angle is considerably different for negative canting angles. These differences are key to the different behavior predicted by the different model sets.

It should also be noted that with ansatz 2, a few terms (which generated complex number solutions) were neglected in the integration of some of these parameters. Specifically, parameter $I_{4}$ had the additional term $-\frac{\sin ^{2} \theta_{c}}{2} \ln \left(-\sin \theta_{c} \cos \theta_{c}\right)$, and parameter $I_{6}$ had the additional term $+\frac{\tan \theta_{c}}{2} \ln \left(-\sin \theta_{c} \cos \theta_{c}\right)$.

Finally, we should note an interesting feature in Eqs. (3)-(6); the ansatz used does not affect the functional form of the model in terms of the collective coordinates. Instead, the effect of the ansatz (including canting) is taken into account in the $I_{i}$ parameters which modulate the strength of the different terms. This is true irrespective of the ansatz used, and can help in extending these models to other Dw structures in the future.

\subsection{Validity of the CCMs}

To assess the accuracy of the collective coordinate models, we initially applied them to a case of current-driven DW motion in $\mathrm{Pt} / \mathrm{CoFe} / \mathrm{MgO}$ under a current density of $J_{x}=0.1 \mathrm{TA} / \mathrm{m}^{2}$ (the same case studied in reference [17]), and field-driven DW motion in $\mathrm{Pt} / \mathrm{Co} / \mathrm{Ni} / \mathrm{Co} / \mathrm{MgO} / \mathrm{Pt}$ under an applied field of $B_{z}=10 \mathrm{mT}$, as outlined in Fig. 4.

In the $\mathrm{Pt} / \mathrm{CoFe} / \mathrm{MgO}$ sample, models with inherent canting (model set 3 ) show superior capability in replicating the micromagnetic results, and require a lower number of degrees of freedom for accurate predictions (only $q$ and $\phi$ ) as highlighted in Fig. 4b. This shows the importance of including canting in the domains when studying samples with lower anisotropy under in-plane fields. As the most accurate models with canting is the $q-\phi$ form of model 3 , the profile used to approximate the DW seems to be more important than adding additional collective coordinates.

In the $\mathrm{Pt} / \mathrm{Co} / \mathrm{Ni} / \mathrm{Co} / \mathrm{MgO} / \mathrm{Pt}$ sample (which has stronger anisotropy and lower DMI, corresponding to smaller canting and tilting), according to Fig. 4(c) we see that the DW tilting has minimal effect on accuracy of the models, while DW width plays an important role in these narrower DWs. We verified this in other models as well, observing that in this case models without canting are better suited to reproduce micromagnetic results (Fig. 4d) likely due to the smaller canting. We also observe that the models are able to predict the starting point and endpoint of the Walker Breakdown behavior properly, and show the right qualitative trends.

In summary, it seems that narrower DWs are better modeled by CCMs that include DW width while for wider DWs this parameter plays a minor role. In addition, in systems with high anisotropy canting effects can be neglected. In the next section, we provide a more detailed analysis of different cases of DW motion under in-plane field using the models verified here.

\section{Results and discussion}

Micromagnetic simulations were performed on the two nanowires outlined in Table 1 with DWs driven by fields or Slonczewski-like spinorbit torques under the applications of longitudinal $\left(B_{x}\right)$ and transverse $\left(B_{y}\right)$ magnetic fields. To interpret the results of the micromagnetic simulations, we used the four time dependent collective coordinates identified earlier.

It is well-known from micromagnetic studies that the motion of the DW reaches steady state conditions after a period of transient behavior, which we also verified for our systems. In our simulations, steady state conditions were reached after about $2.5 \mathrm{~ns}$ in most cases, with $\dot{\phi} \sim \dot{\chi} \sim \dot{\Delta} \sim 0$. While we found the evolution of the micromagnetic model to not exactly match the CCMs, a steady state condition was identified in the CCMs as well. In CCMs without the tilting of the DW, a steady state condition was observed with $\dot{\phi} \sim \dot{\Delta} \sim 0$, while in tilted models we found only $\dot{\Delta} \sim 0$ (although in many cases $\dot{\phi} \sim \dot{\chi}<1$ ). In steady state conditions, the collective coordinate models may be simplified to better understand the critical points which can be identified in the micromagnetic simulations. In this section we use a ${ }^{*}$ to denote steady state values of the collective coordinate.

In the next subsections, we will show which CCMs were able to better predict the micromagnetic results for different combinations of in-plane fields and drive interactions, and use these models to highlight features or critical points in the dynamics of the DW. By better predicting the micromagnetic results, we mean reproducing the results with the lowest error over a wider range of fields. Note that the range of in-plane field values over which different collective coordinate models can be solved with a convergent solution is different for different materials and drive-conditions; we only show cases where a solution could be calculated.

\subsection{General observations}

We identified several general features in the simulations. First, as outlined in our previous work [17], one notes that domains under large in-plane fields can no longer be assumed to be fully perpendicularly magnetized, but clearly show some canting of the magnetization into the plane of the sample. This effect was much smaller in $\mathrm{Pt} / \mathrm{Co} / \mathrm{Ni} / \mathrm{Co} / \mathrm{MgO} / \mathrm{Pt}$ compared to $\mathrm{Pt} / \mathrm{CoFe} / \mathrm{MgO}$, due mainly to the difference in the uniaxial magnetic anisotropy of the two samples.

Second, in the $\mathrm{Pt} / \mathrm{Co} / \mathrm{Ni} / \mathrm{Co} / \mathrm{MgO} / \mathrm{Pt}$ sample we observed limited tilting of the DW (only up to 10 degrees in many cases) which likely is due to the much lower DMI compared to the $\mathrm{Pt} / \mathrm{CoFe} / \mathrm{MgO}$ sample. As a result, we expect the $\chi$ coordinate to play a small role in modeling this system.

Third, we found that DW shape and rigidity (lack of elasticity) are affected by in-plane fields. As depicted in Fig. 5, depending on the combination of drive interaction and in-plane fields, the DW might have a rigid line shape, or a curved shape (either S-shaped or an arch of a circle). With large in-plane fields (longitudinal and transverse), the DW 


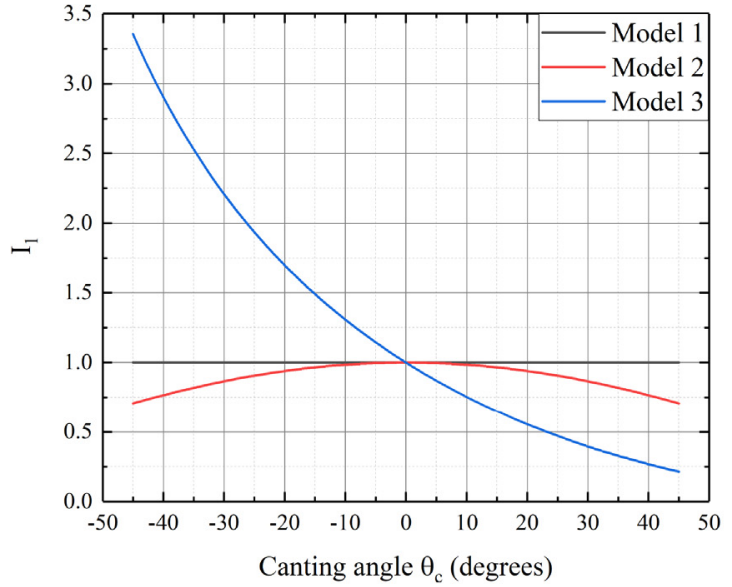

(a) $I_{1}$.

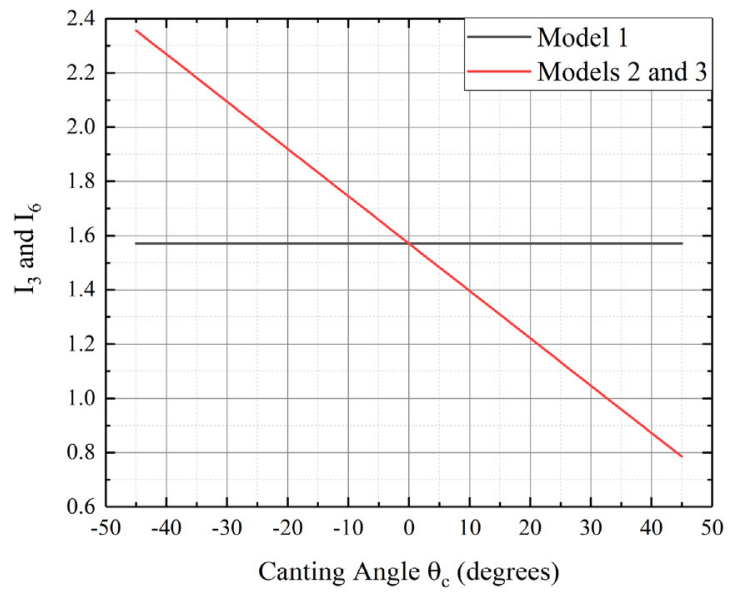

(c) $\mathrm{I}_{3}$.

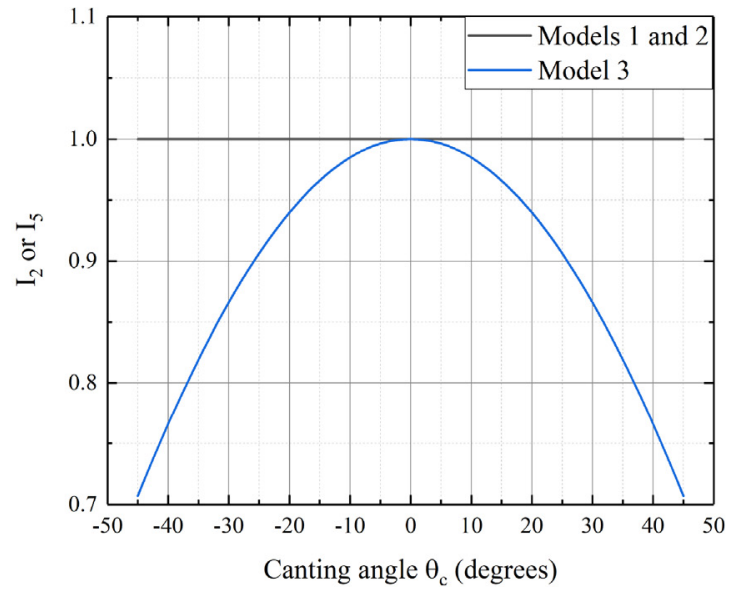

(b) $\mathrm{I}_{2}$.

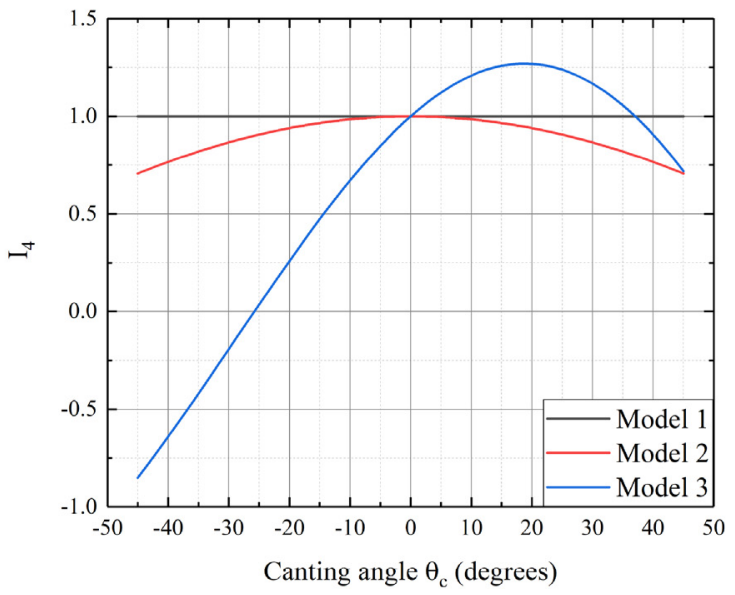

(d) $\mathrm{I}_{4}$.

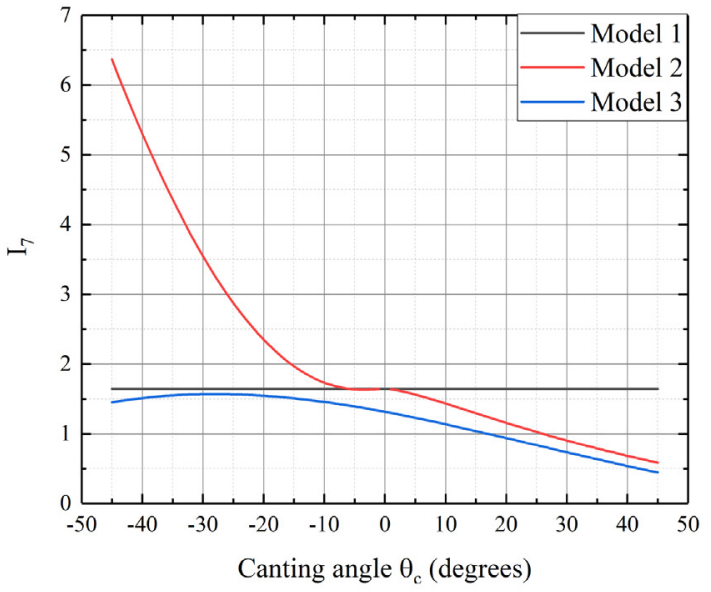

(e) $\mathrm{I}_{7}$.

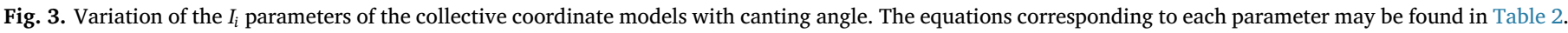

might lose its rigidity, and instead extend elastically through the system. In $\mathrm{Pt} / \mathrm{CoFe} / \mathrm{MgO}$ system, we found both longitudinal and transverse fields where the DW starts to elongate instead of moving rigidly. Thess fields were dependent on the material properties, and also the driving interaction applied to the system. In the $\mathrm{Pt} / \mathrm{Co} / \mathrm{Ni} / \mathrm{Co} / \mathrm{MgO} / \mathrm{Pt}$ system, these effects were not observed, likely due to the high uniaxial anisotropy of the system which helps maintain the DW shape. However, in this material the DW shape was disrupted due to other features which will be discussed later.

\subsection{Domain wall motion under longitudinal in-plane fields}

\subsubsection{Field-driven case}

The two samples were studied under drive fields of $B_{z}=5 \mathrm{mT}, 30 \mathrm{mT}$. Longitudinal in-plane fields in the range $B_{x}=-225 m T$ to $325 \mathrm{mT}$ were used for $\mathrm{Pt} / \mathrm{CoFe} / \mathrm{MgO}$, while a range of $B_{x}=-500 \mathrm{mT}$ to $500 \mathrm{mT}$ was used for $\mathrm{Pt} / \mathrm{Co} / \mathrm{Ni} / \mathrm{Co} / \mathrm{MgO} / \mathrm{Pt}$. The results of these micromagnetic simulations are presented in Figs. 7 and 8, and compared to the most accurate collective coordinate models. 


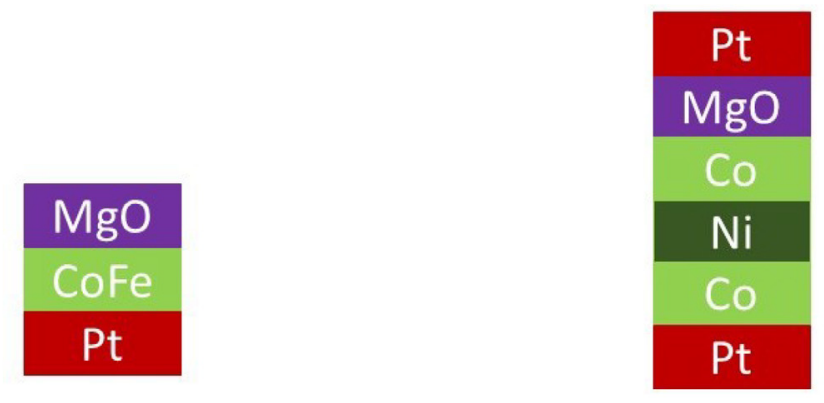

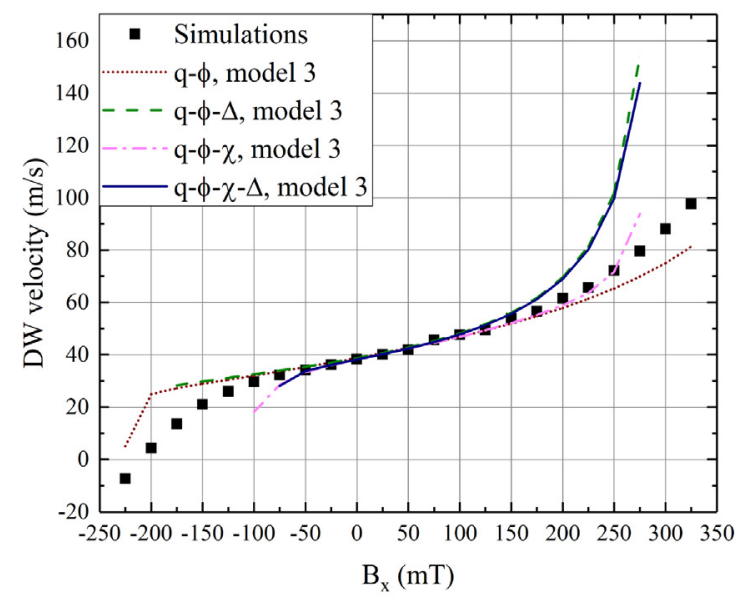

(a) Model 3 for $\mathrm{Pt} / \mathrm{CoFe} / \mathrm{MgO}$.

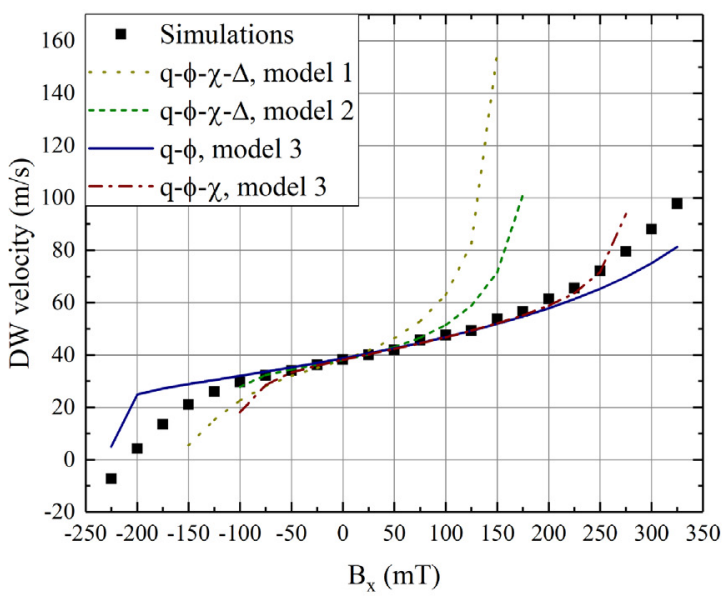

(c) Most accurate models for $\mathrm{Pt} / \mathrm{CoFe} / \mathrm{MgO}$.

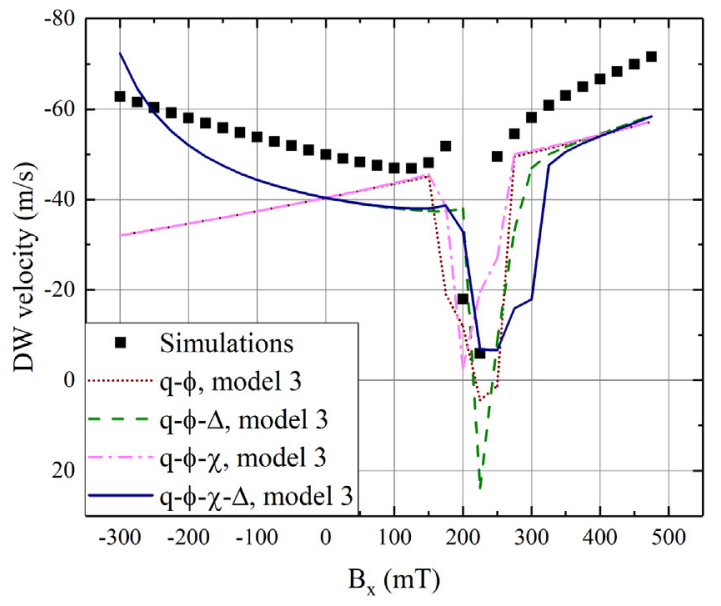

(b) Model 3 for $\mathrm{Pt} / \mathrm{Co} / \mathrm{Ni} / \mathrm{Co} / \mathrm{MgO} / \mathrm{Pt}$.

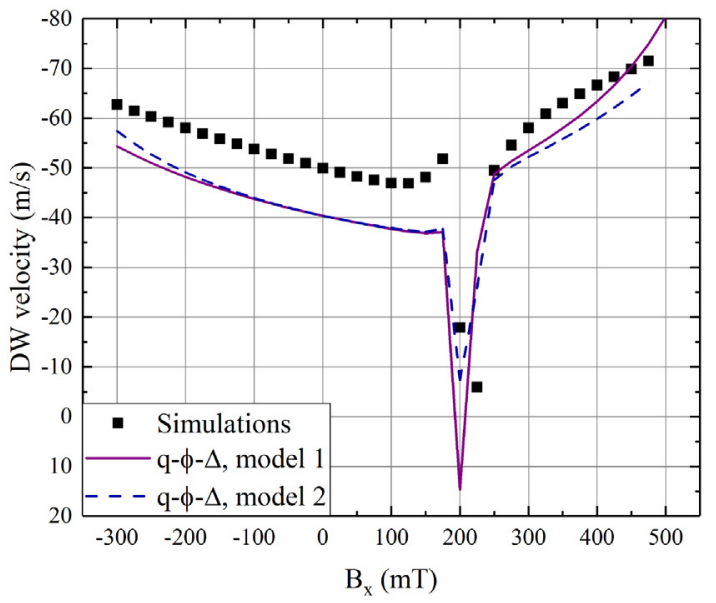

(d) Most accurate models for $\mathrm{Pt} / \mathrm{Co} / \mathrm{Ni} / \mathrm{Co} / \mathrm{MgO} / \mathrm{Pt}$.

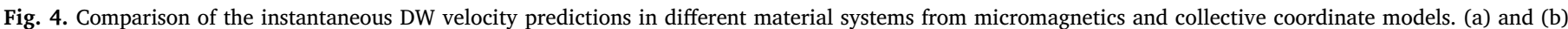

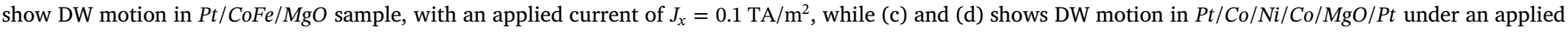

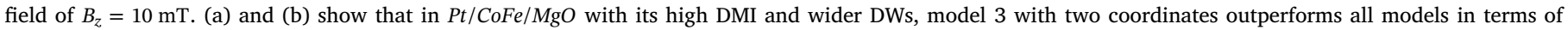

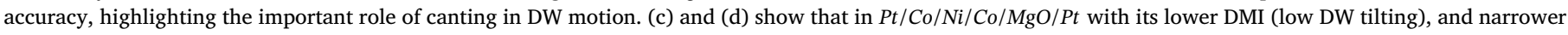

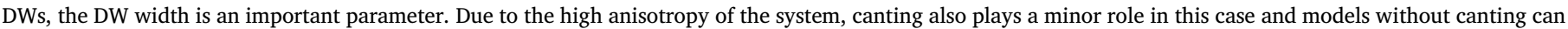

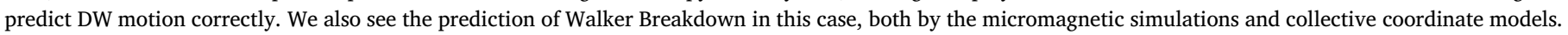

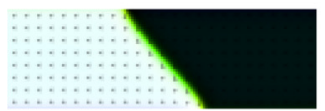

(a) $\mathrm{B}_{\mathrm{x}}=-200 \mathrm{mT}$

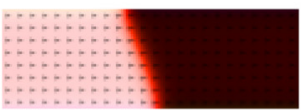

(b) $\mathrm{B}_{\mathrm{x}}=300 \mathrm{mT}$

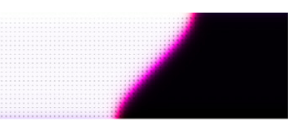

(c) $\mathrm{B}_{\mathrm{y}}=-100 \mathrm{mT}$

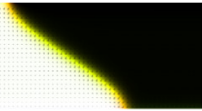

(d) $\mathrm{B}_{\mathrm{y}}=100 \mathrm{mT}$

Fig. 5. Snapshots of different shapes of the DWs observed under a current density of $J_{x}=0.1 \mathrm{TA} / \mathrm{m}^{2}$ in $P t / \mathrm{CoFe} / \mathrm{MgO}$ after 5 ns. (a) and (b) show a rigidly moving linear DW, while the DW in (c) and (d) is rather S-shaped. 


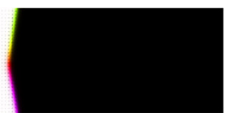

(a) $\mathrm{B}_{\mathrm{z}}=10 \mathrm{mT}, \mathrm{B}_{\mathrm{x}}=225 \mathrm{mT}$.

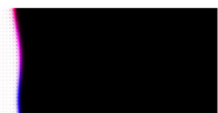

(b) $\mathrm{B}_{\mathrm{z}}=30 \mathrm{mT}, \mathrm{B}_{\mathrm{x}}=225 \mathrm{mT}$

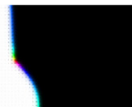

(c) $\mathrm{B}_{\mathrm{z}}=30 \mathrm{mT}, \mathrm{B}_{\mathrm{x}}=100 \mathrm{mT}$

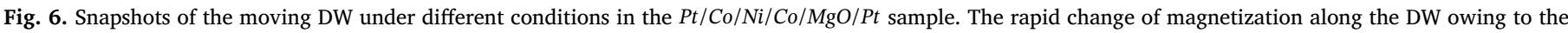
Walker Breakdown can be observed.

Comparing the variation of velocity for the two samples, as depicted in Figs. $7 \mathrm{a}$ and $8 \mathrm{a}$, we see that in both cases the general trend with the drive field is the same; the velocity and nonlinearity of the curve increases with increasing drive field $\left(B_{z}\right)$, while changing $B_{x}$ tunes the velocity to an extent (with the curve having a minimum with respect to the longitudinal field). The DW velocity predictions are qualitatively in agreement the behavior observed in experiments [46,47]. However, the $\mathrm{Pt} / \mathrm{Co} / \mathrm{Ni} / \mathrm{Co} / \mathrm{MgO} / \mathrm{Pt}$ sample shows the additional effect of a sudden drop in DW velocity for a range of in-plane fields applied. This Walker Breakdown (WB) like behavior [48] was verified by looking at the snapshots of the moving DW (depicted in Fig. 6), where we can see local precession of the magnetization and formation of vertical Bloch lines arising from the edge that modify the DW structure [32]. This behavior could be attributed to the higher anisotropy of this material, which reduces the local field needed to reach WB. Note that this behavior is local; the DW does not oscillate back and forth as a single object (unlike an actual WB behaviour during which the DW moves back and fourth rapidly), but the overall effect of the local precession of magnetization over time is equivalent to the DW moving back and forth rigidly and slowing down, which is why the collective coordinate model can replicate this effect to an extent.

In terms of the CCMs, we found models without canting to better reproduce the results for $\mathrm{Pt} / \mathrm{Co} / \mathrm{Ni} / \mathrm{Co} / \mathrm{MgO} / \mathrm{Pt}$ (where canting and DW tilting are small). However, in this material the DW width parameter $\Delta$ was important in predicting the DW behavior properly. In $\mathrm{Pt} / \mathrm{CoFe} / \mathrm{MgO}$ with its higher canting and tilting of the DW, we found that $q-\phi$ form of model 3 (with inherent canting) is better suited in predicting the DW behavior.

Looking closely at Figs. 7b and 8b, we find a serious flaw in model set 3 ; this model set seems to not be able to predict the DW width correctly, which in turn can affect its outputs. As such, when a two coordinate form of this model is used, it is able to better predict the DW motion. This also shows why this model set is not suitable for the $\mathrm{Pt} / \mathrm{Co} / \mathrm{Ni} / \mathrm{Co} / \mathrm{MgO} / \mathrm{Pt}$ sample where lack of canting and tilting mean $\Delta$ is one of the main variables affecting the DW. Overall, this observation suggests that the $q-\phi$ form of model 3 is the most suitable for studying DW motion in these systems.

A major difference between the two cases can be observed in the DW's tilting behavior; while $\mathrm{Pt} / \mathrm{CoFe} / \mathrm{MgO} \mathrm{DWs}$ always maintain a positive tilting, in the case of $\mathrm{Pt} / \mathrm{Co} / \mathrm{Ni} / \mathrm{Co} / \mathrm{MgO} / \mathrm{Pt}$ negative tilting can be observed which is likely due to the lower DMI strengths and the higher applied fields used (see Figs. 7e and 8e). Another notable feature of the DW behavior could be seen in Fig. 7c where at a specific field $\phi-\chi \sim 0$ independent of the drive field, while in Fig. $8 \mathrm{c}$ a point could be observed for which $\phi-\chi \sim \frac{\pi}{2}$. We label these points as critical in-plane fields which will be discussed in details in later sections.

\subsubsection{Current-driven case}

Figs. 9 and 10 illustrate the results of micromagnetic simulations for current-driven DW motion under longitudinal fields. The trends observed in the velocity of current-driven DW motion (Figs. 9a and 10a) are in general agreement with published experimental results $[27,28,49,50]$. A somewhat linear behavior is observed for low longitudinal fields, which becomes non-linear as the in-plane field increases. The non-linearity in behavior also seems to increase with increasing current. In terms of the CCMs, we saw results similar to the field-driven case, with models without canting being more suitable for the high anisotropy system and those with canting more suitable for the low anisotropy high DMI system.

We also observe a point where the DW velocity is zero in both cases; the in-plane field at which this happens is another critical point of interest. For the system with lower DMI the nonlinearity in the DW velocity seems to be observable mainly around this point, while in the system with larger DMI this nonlinear behavior is observed over all inplane fields studied. Interestingly, this in-plane field seems to have an additional feature: the DW will have the same tilting angle $\chi$ for different drive interactions ((Figs. 9e and 10e)). In the system with the higher anisotropy and lower DMI (namely $\mathrm{Pt} / \mathrm{Co} / \mathrm{Ni} / \mathrm{Co} / \mathrm{MgO} / \mathrm{Pt}$ ), we also observe that at this point $\phi-\chi \sim 90^{\circ}$ (a fully Bloch DW).

One unexpected result was the presence of Walker Breakdown in our initial current-driven simulations of $\mathrm{Pt} / \mathrm{Co} / \mathrm{Ni} / \mathrm{Co} / \mathrm{MgO} / \mathrm{Pt}$; however, in these cases while a vertical Bloch line is nucleated, it is shortlived and simulating for longer durations shows that this is just a transitory effect. One point with such an effect can be observed in Fig. $10 \mathrm{a}$ as an outlier at $B_{x}=250 \mathrm{mT}$.

In terms of angles, an in-plane field exists for which $\phi-\chi \sim 0$ (Figs. 9c and 10c); in the $\mathrm{Pt} / \mathrm{CoFe} / \mathrm{MgO}$ sample we also see a case of $\phi=0$ (Figs. 9d) which is absent in the $\mathrm{Pt} / \mathrm{Co} / \mathrm{Ni} / \mathrm{Co} / \mathrm{MgO} / \mathrm{Pt}$ sample. These could be points of interest for further analytical studies.

\subsection{Domain wall motion under transverse fields}

Figs. 11 and 12 show the results of field-driven DW motion under the application of transverse fields in the materials under study, while Figs. 13 and 14 show the results of current-driven DW motion under transverse fields.

As observed from Figs. 11e, 12e, 13e, and 14e, the DW tilting angle changes dramatically under transverse fields with a behavior different compared to what was observed under longitudinal fields. While under longitudinal fields we only observed positive or negative tilting for a specific material, under transverse fields we can observe both types of angles; in a sense the transverse fields could be used to control the tilting of the DW. Obviously, under these conditions the tilting of the DW is an important coordinate. Yet we see that the collective coordinate models are accurate in predicting the behavior of the DW, with and without the tilting included in the models.

In the $\mathrm{Pt} / \mathrm{CoFe} / \mathrm{MgO}$ sample (Figs. 11c, and 13c), we can identify a critical transverse fields at which $\phi \sim \chi \sim 0$. In the $\mathrm{Pt} / \mathrm{Co} / \mathrm{Ni} / \mathrm{Co} / \mathrm{MgO} / \mathrm{Pt}$ system (Figs. 12, and 14 panels c, d, and e), we instead have a point where $\chi=0$ and $\phi=180$. These points coincide with when the DW is fully Néel, with the difference in magnetization being due to the different chirality of the DW in the two systems.

\subsection{Analysis of the critical points}

In the micromagnetic simulations and collective coordinate results of Figs. 7-14, we were able to identify several points where the DW behavior showed features that could be reproduced irrespective of material properties. These points could be used to derive simplified forms of the DW dynamic equations. 


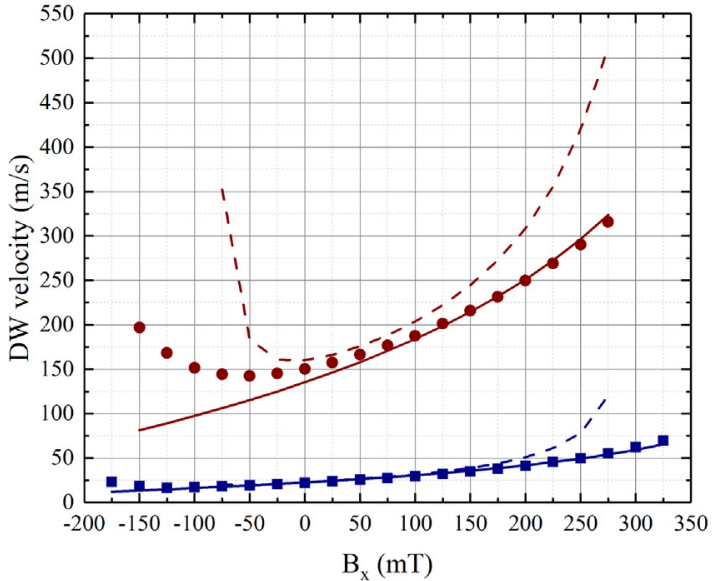

(a) DW velocity.

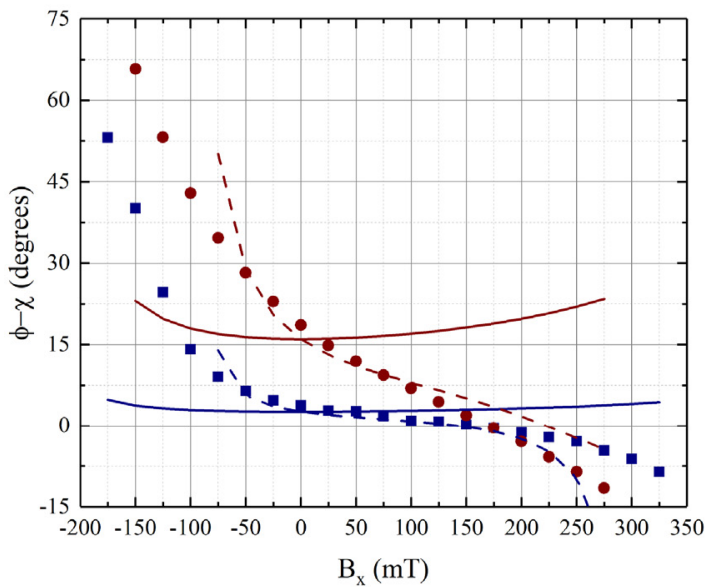

(c) $\phi-\chi$

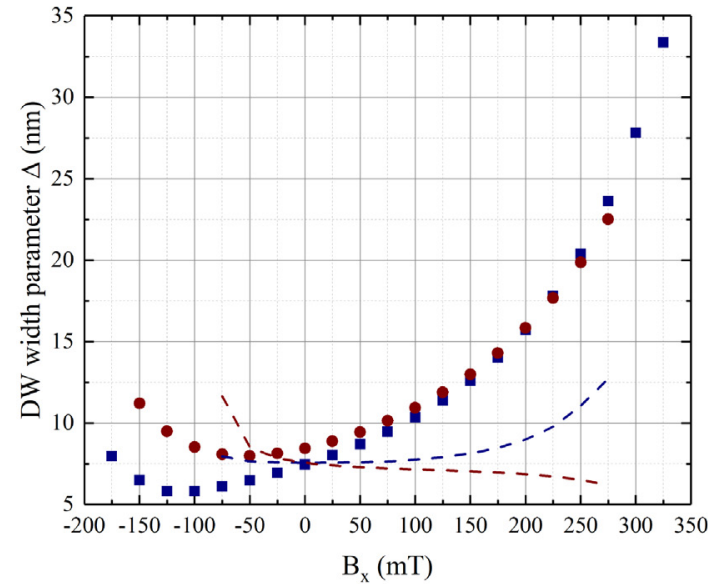

(b) DW width parameter $(\Delta)$.

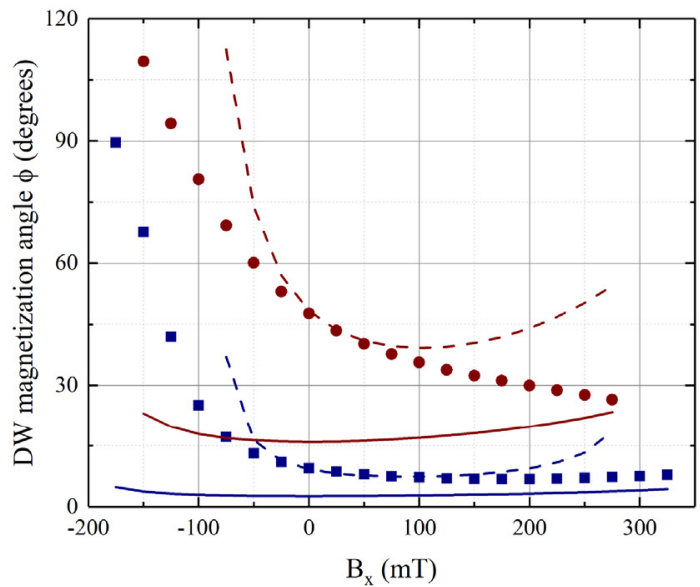

(d) DW magnetization angle $(\phi)$.

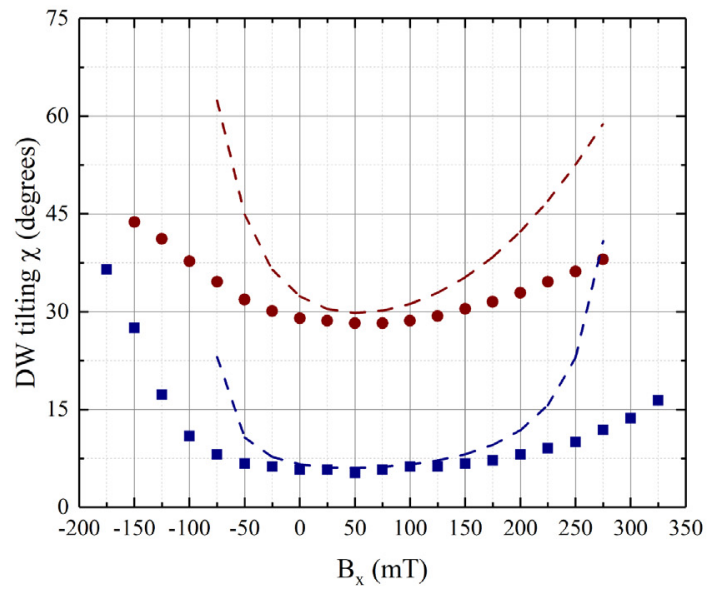

- $\mathrm{B}_{\mathrm{z}}=5 \mathrm{mT}, \mu \mathrm{mag}$

- $\mathrm{B}_{\mathrm{z}}=30 \mathrm{mT}, \mu \mathrm{mag}$

- - $\mathrm{B}_{\mathrm{z}}=5 \mathrm{mT}, \mathrm{q}-\phi-\chi-\Delta$ model 3

- - $\mathrm{B}_{\mathrm{z}}=30 \mathrm{mT}$, $\mathrm{q}-\phi-\chi-\Delta \operatorname{model} 3$

$-\mathrm{B}_{\mathrm{z}}=5 \mathrm{mT}$, q- $\phi$ model 3

$\mathrm{B}_{\mathrm{z}}=30 \mathrm{mT}$, q- $\phi$ model 3

(e) DW tilting angle $(\chi)$

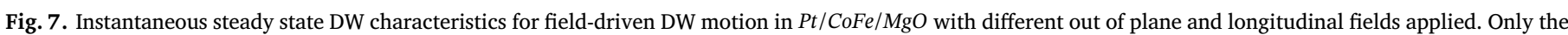
collective coordinate models with highest accuracy in predicting the DW velocity are shown.

We observed that in several cases (field- and current-driven DW motion under longitudinal and transverse fields in $\mathrm{Pt} / \mathrm{CoFe} / \mathrm{MgO}$, and field-driven case under transverse fields and field- and current-driven cases under transverse field for $\mathrm{Pt} / \mathrm{Co} / \mathrm{Ni} / \mathrm{Co} / \mathrm{MgO} / \mathrm{Pt}$ ), an in-plane field exists for which $\phi-\chi \sim 0$ or $180^{\circ}$. In addition, under longitudinal fields this point is independent of the drive interaction. We observed in Figs. 7-14 that the CCMs can accurately predict the DW behavior at this point. From a CCM perspective, this is the point where the DW has a fully Néel like structure. This means the contributions from the DMI and magnetostatic terms become zero, and we have: 


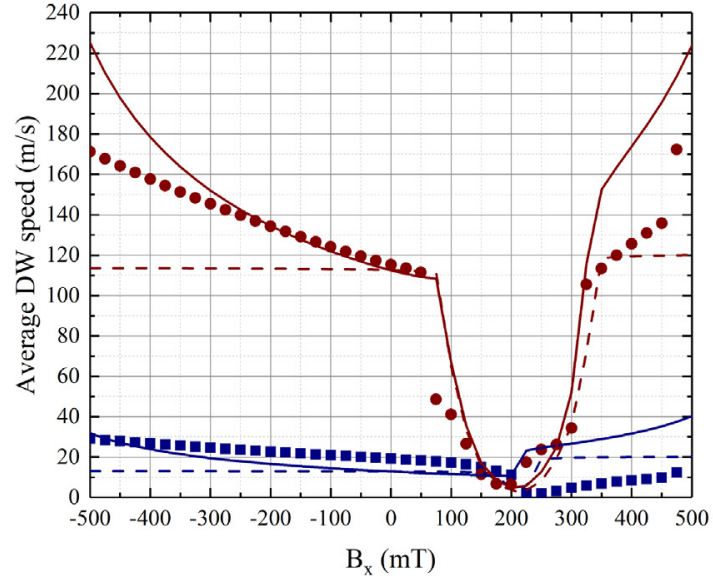

(a) DW average speed.

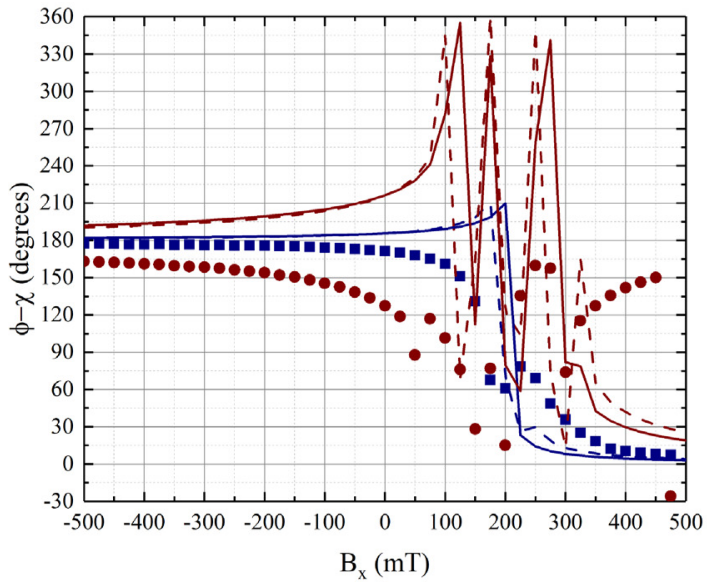

(c) Instantaneous $\phi-\chi$

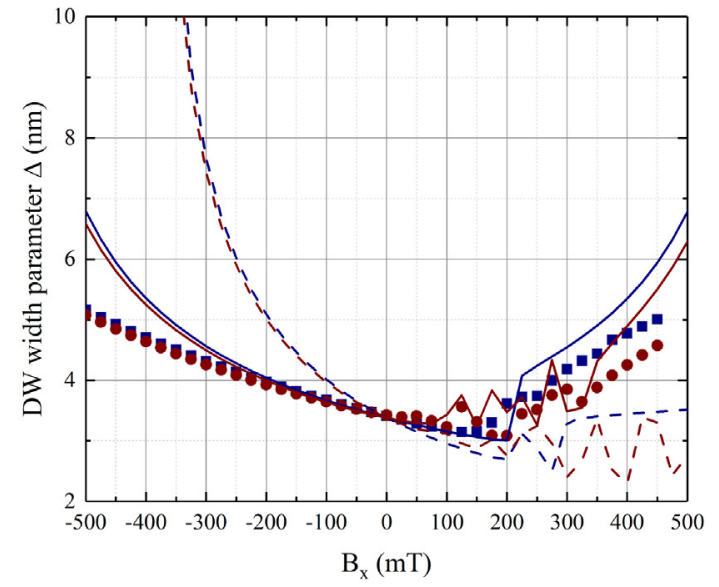

(b) Instantaneous DW width parameter $(\Delta)$.

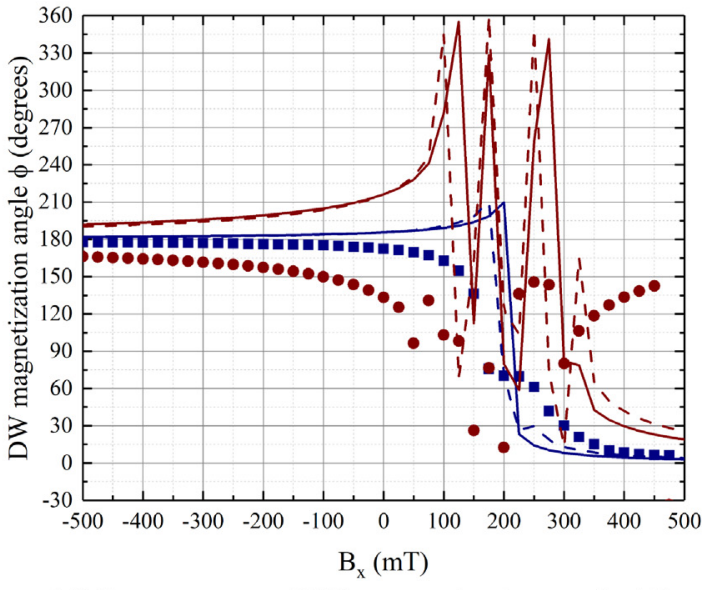

(d) Instantaneous DW magnetization angle $(\phi)$.
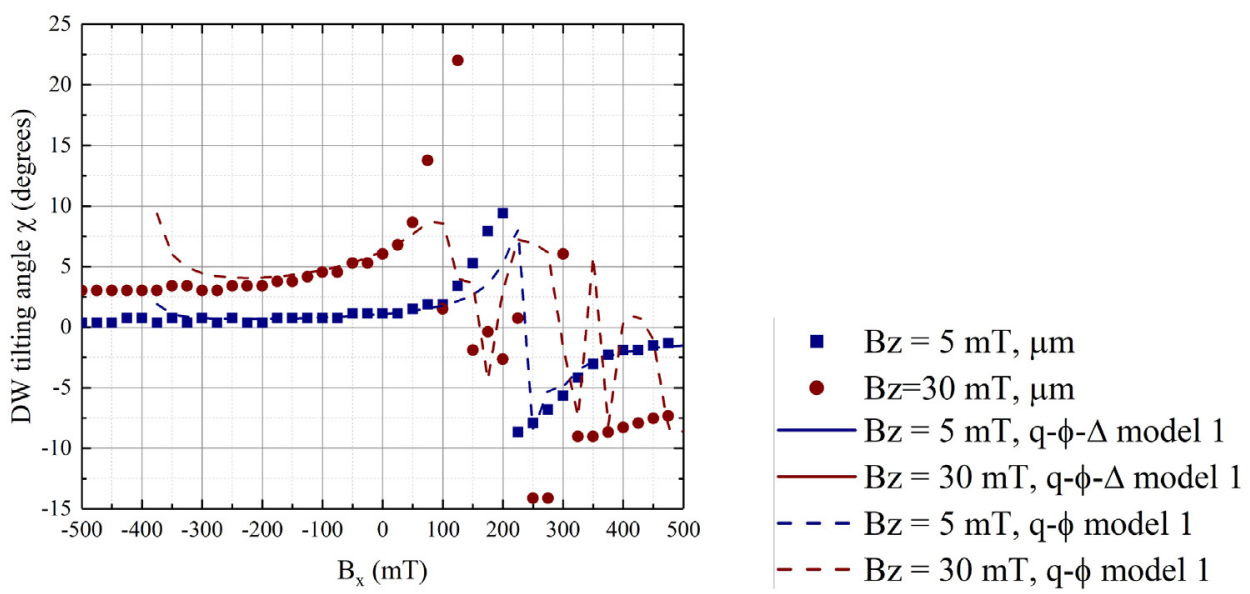

(e) Instantaneous DW tilting angle $(\chi)$.

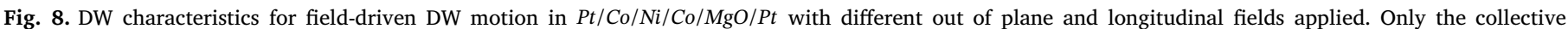

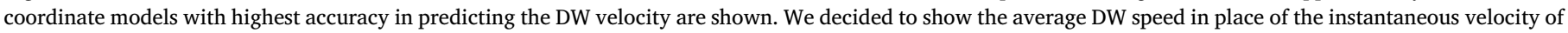
the DW, due to the walker breakdown behavior; this behavior is observed in panels (b)-(e) for $50 \mathrm{mT}<B_{x}<300 \mathrm{mT}$. 


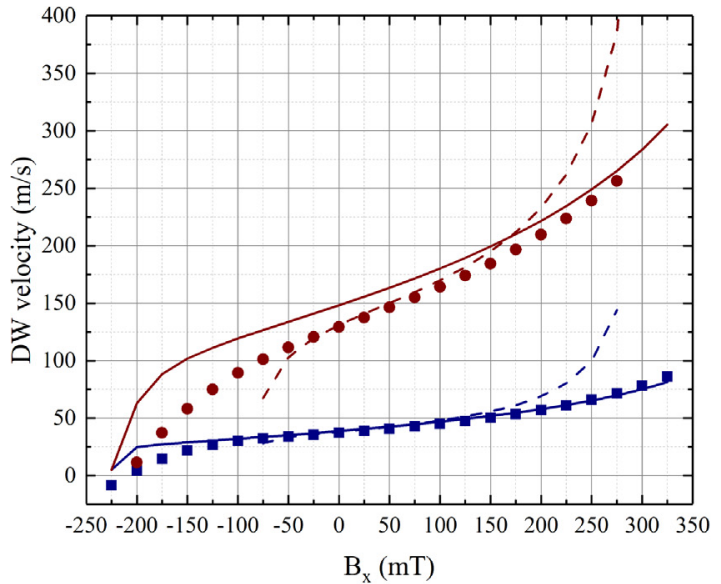

(a) DW velocity.

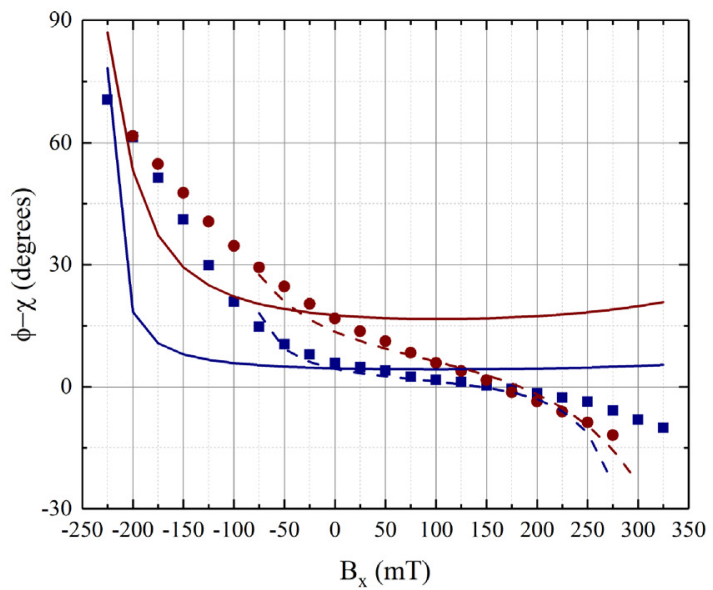

(c) $\phi-\chi$

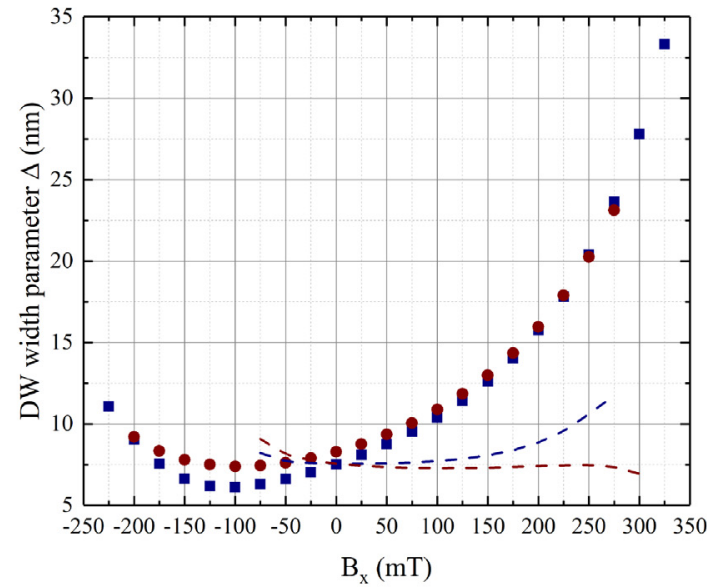

(b) DW width parameter $(\Delta)$.

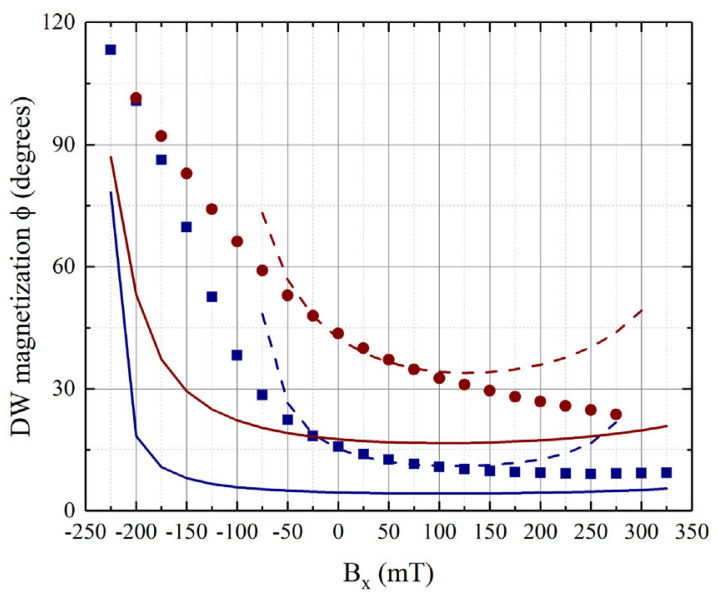

(d) DW magnetization angle $(\phi)$.

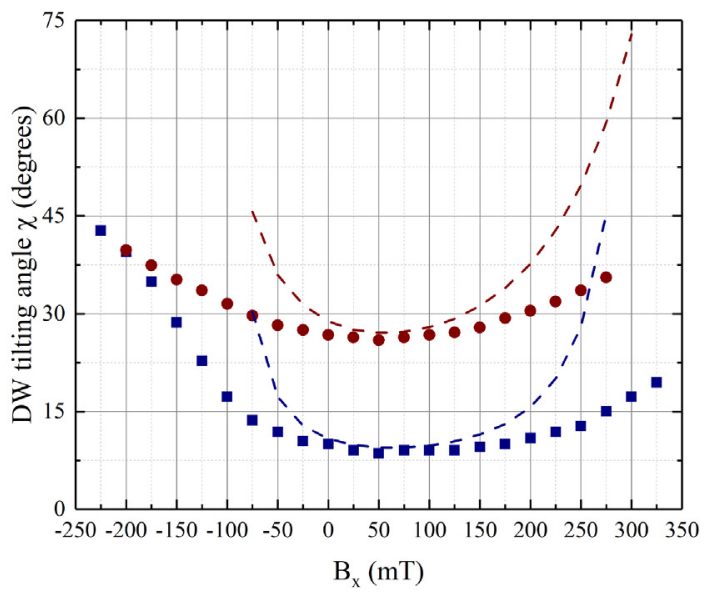

- $\mathrm{J}=0.1 \mathrm{TA} / \mathrm{m}^{2}, \mu \mathrm{mag}$

- $\mathrm{J}=0.4 \mathrm{TA} / \mathrm{m}^{2}, \mu \mathrm{mag}$

- - $\mathrm{J}=0.1 \mathrm{TA} / \mathrm{m}^{2}, \mathrm{q}-\phi-\chi-\Delta$ model 3

- - $\mathrm{J}=0.4 \mathrm{TA} / \mathrm{m}^{2}, \mathrm{q}-\phi-\chi-\Delta \operatorname{model} 3$

$-\mathrm{J}=0.1 \mathrm{TA} / \mathrm{m}^{2}, \mathrm{q}-\phi$ model 3

$\mathrm{J}=0.4 \mathrm{TA} / \mathrm{m}^{2}, \mathrm{q}-\phi$ model 3

(e) DW tilting angle $(\chi)$.

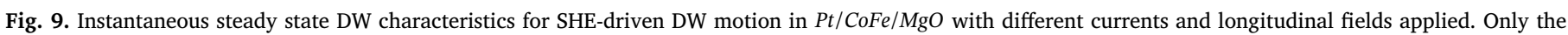
collective coordinate models with highest accuracy in predicting the DW velocity are shown. 


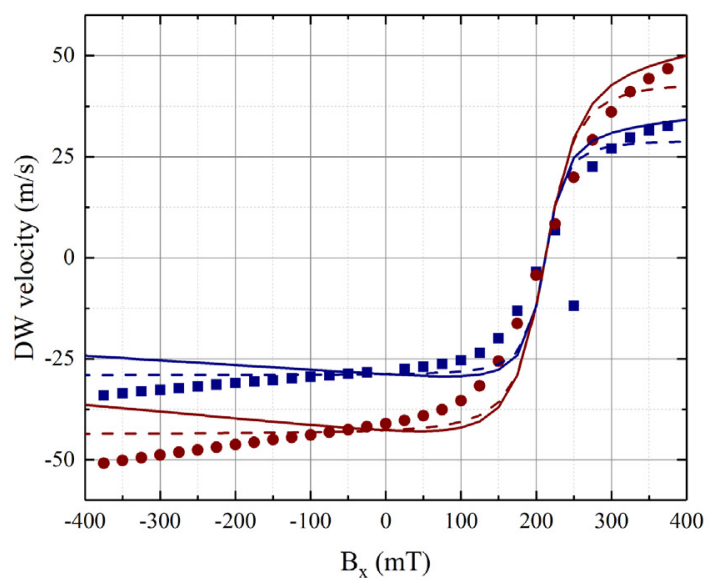

(a) DW velocity.

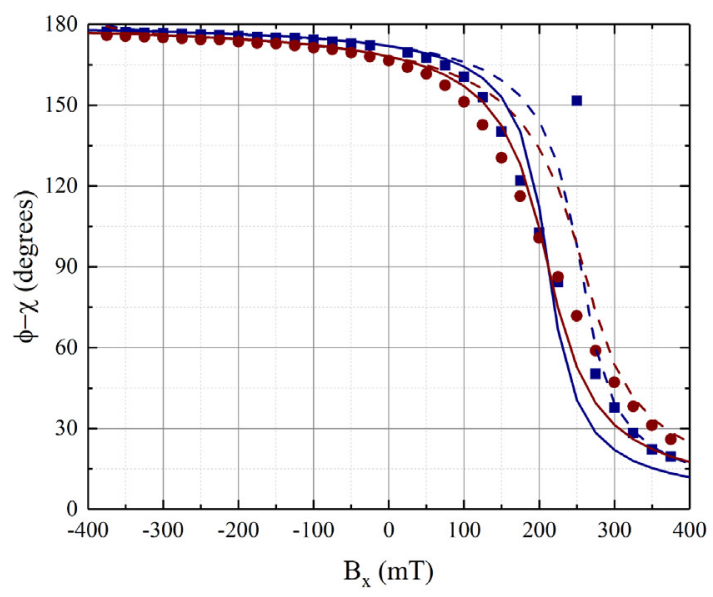

(c) $\phi-\chi$

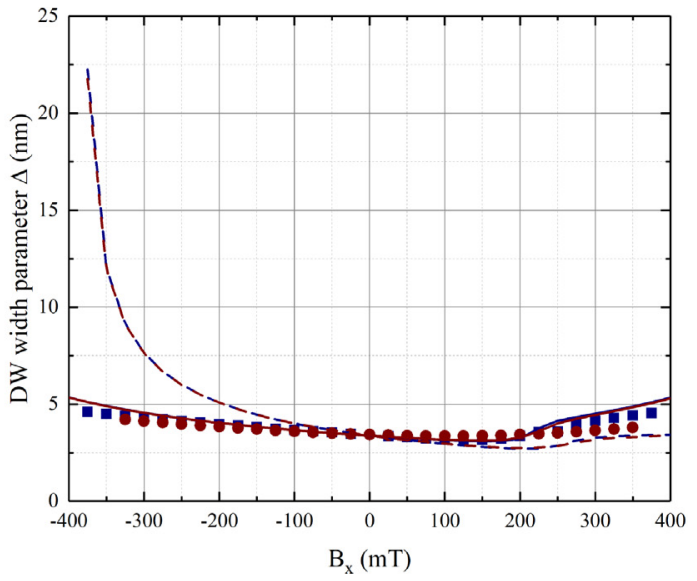

(b) DW width parameter $(\Delta)$.

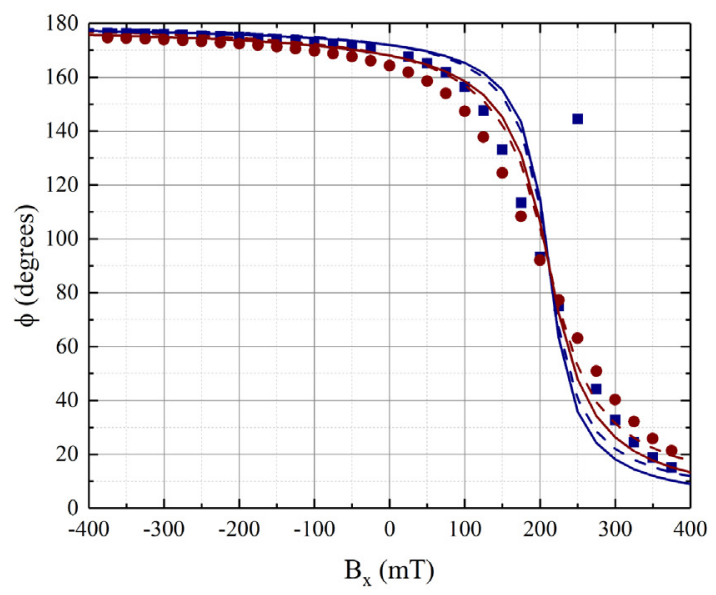

(d) DW magnetization angle $(\phi)$.

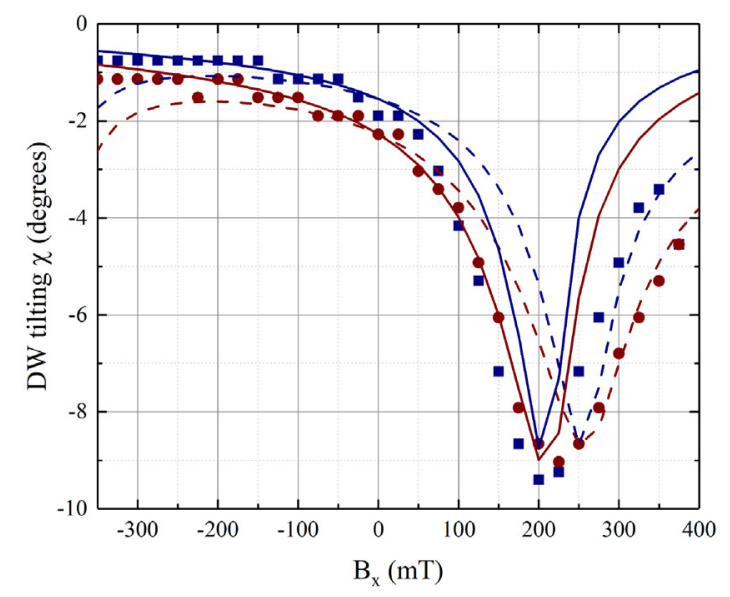

- $\mathrm{J}=0.1 \mathrm{TA} / \mathrm{m}^{2}, \mu \mathrm{mag}$

- $\mathrm{J}=0.15 \mathrm{TA} / \mathrm{m}^{2}, \mu \mathrm{mag}$ $\mathrm{J}=0.1 \mathrm{TA} / \mathrm{m}^{2}$, q- $\phi$ model 3 $\mathrm{J}=0.15 \mathrm{TA} / \mathrm{m}^{2}, \mathrm{q}-\phi$ model 3 $---\mathrm{J}=0.1 \mathrm{TA} / \mathrm{m}^{2}$, q- $\phi$ model 1

(e) DW tilting angle $(\chi)$.

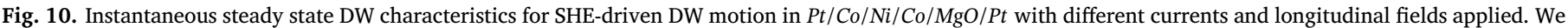

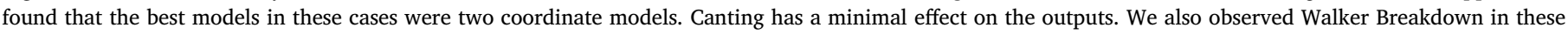
cases. Only the collective coordinate models with highest accuracy in predicting the DW velocity are shown. 


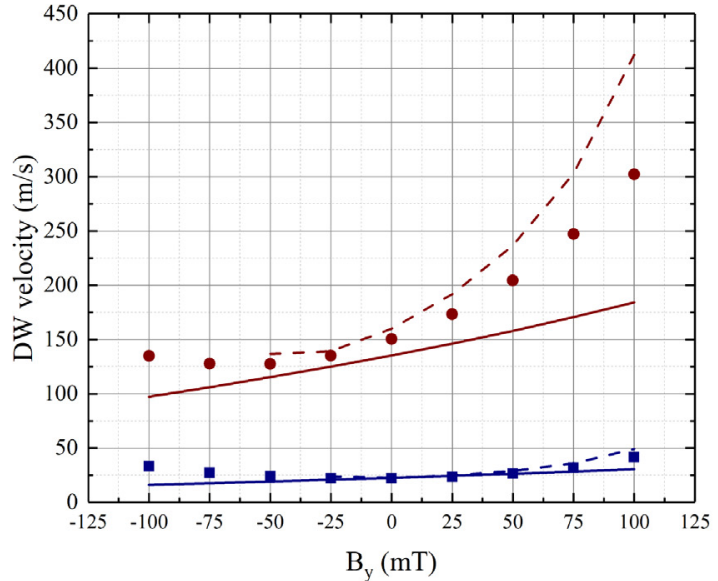

(a) DW velocity.

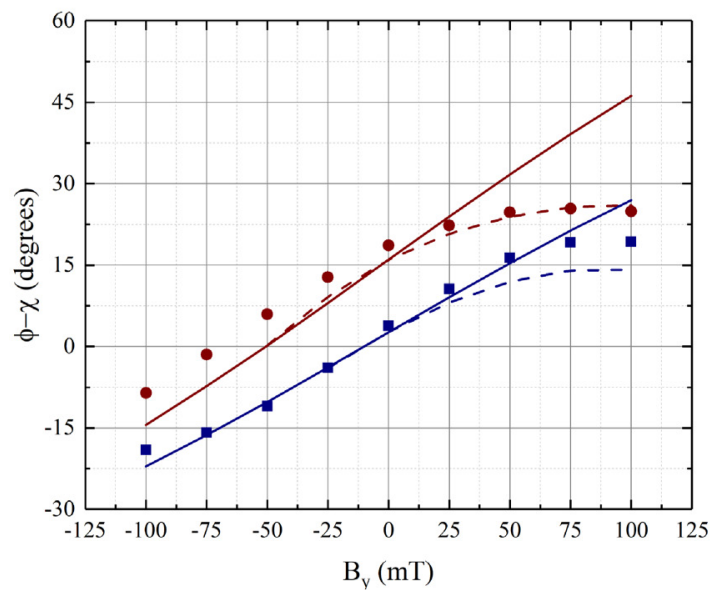

(c) $\phi-\chi$

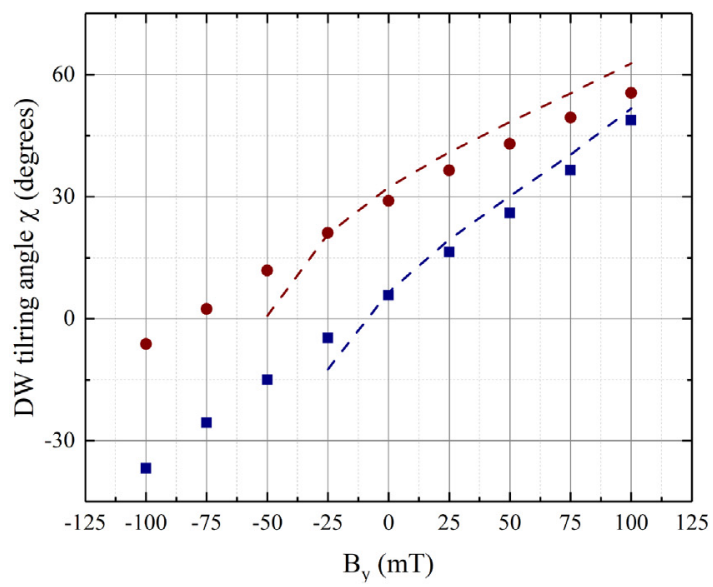

(e) DW tilting angle $(\chi)$.

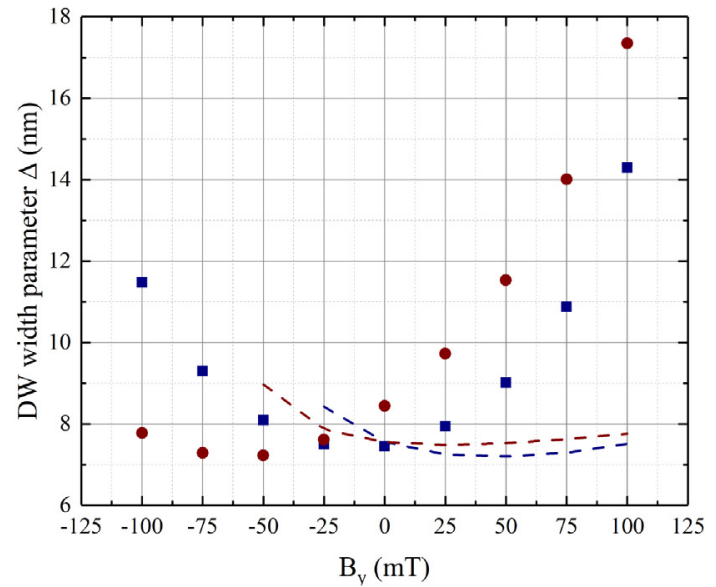

(b) DW width parameter $(\Delta)$.

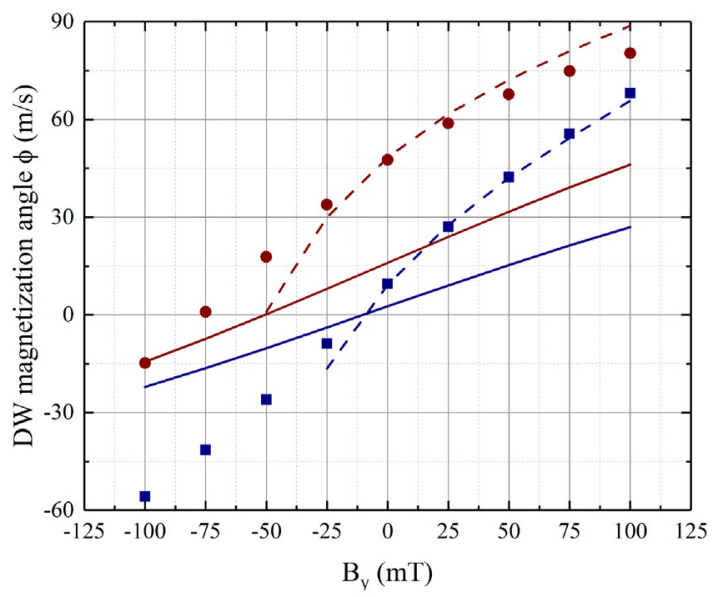

(d) DW magnetization angle $(\phi)$.

$$
\begin{aligned}
\mathrm{B}_{\mathrm{z}} & =5 \mathrm{mT}, \mu \mathrm{mag} \\
\mathrm{B}_{\mathrm{z}} & =30 \mathrm{mT}, \mu \mathrm{mag} \\
--\mathrm{B}_{\mathrm{z}} & =5 \mathrm{mT}, \mathrm{q}-\phi-\chi-\Delta \text { model } 3 \\
---\mathrm{B}_{\mathrm{z}}= & 30 \mathrm{mT}, \mathrm{q}-\phi-\chi-\Delta \text { model } 3 \\
-\mathrm{B}_{\mathrm{z}}= & 5 \mathrm{mT}, \mathrm{q}-\phi \text { model } 3 \\
-\mathrm{B}_{\mathrm{z}} & =30 \mathrm{mT}, \mathrm{q}-\phi \text { model } 3
\end{aligned}
$$

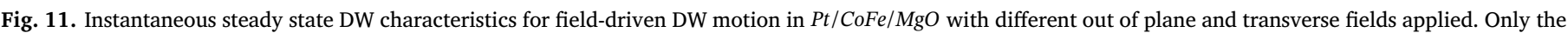
collective coordinate models with highest accuracy in predicting the DW velocity are shown. 


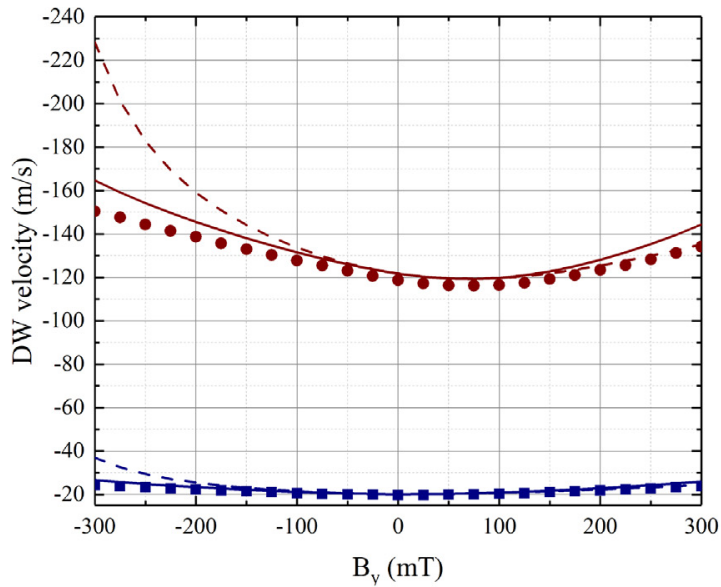

(a) DW velocity.

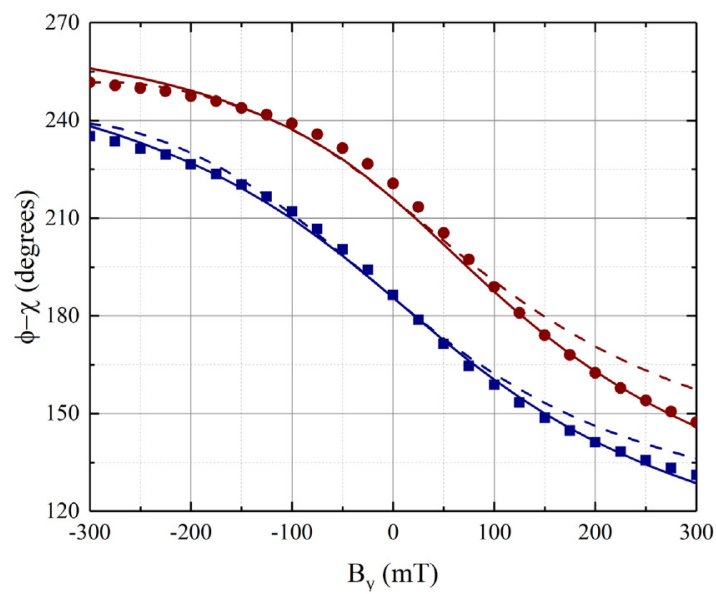

(c) $\phi-\chi$

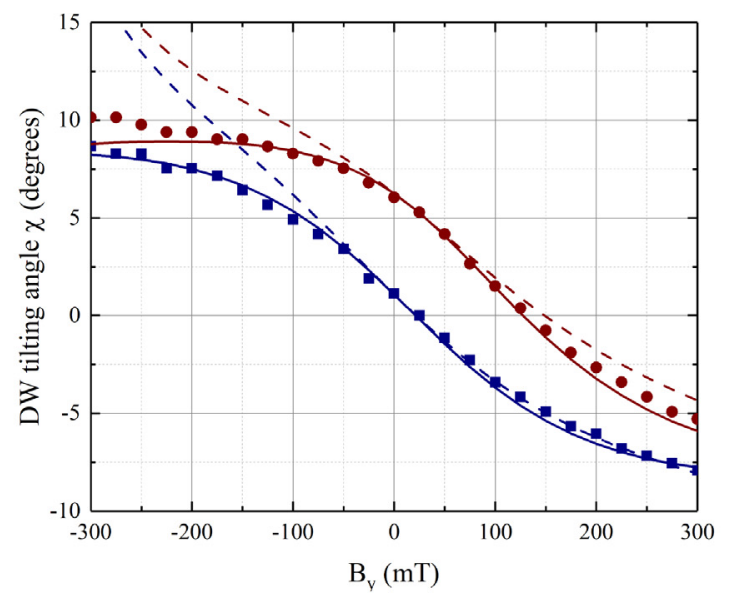

(e) DW tilting angle $(\chi)$.

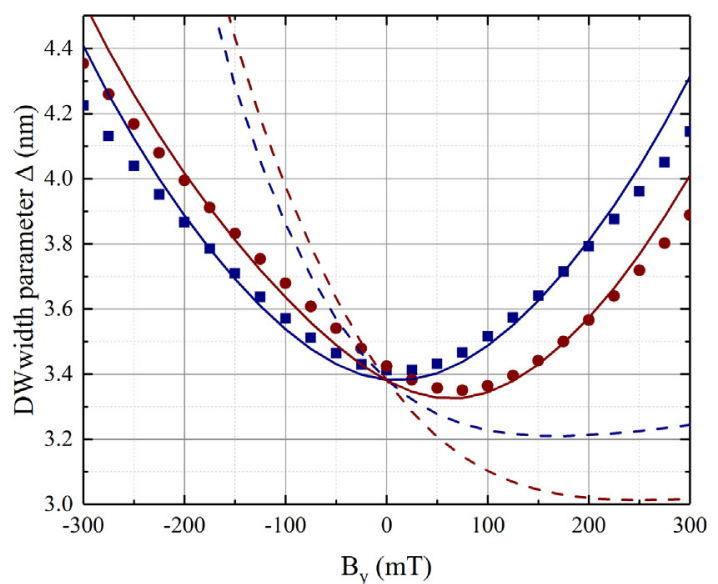

(b) DW width parameter $(\Delta)$.

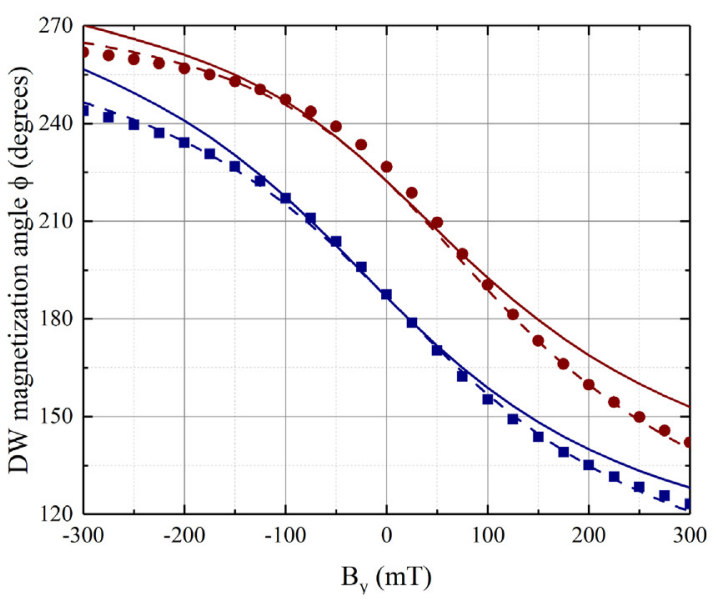

(d) DW magnetization angle $(\phi)$.

- $\mathrm{Bz}=5 \mathrm{mT}, \mu \mathrm{mag}$

- $\mathrm{Bz}=30 \mathrm{mT}, \mu \mathrm{mag}$

- - $\mathrm{Bz}=5 \mathrm{mT}, \mathrm{q}-\phi-\chi-\Delta$ model 3

- - $\mathrm{Bz}=30 \mathrm{mT}, \mathrm{q}-\phi-\chi-\Delta$ model 3

$-\mathrm{Bz}=5 \mathrm{mT}, \mathrm{q}-\phi-\chi-\Delta$ model 1

$\mathrm{Bz}=30 \mathrm{mT}, \mathrm{q}-\phi-\chi-\Delta$ model 1

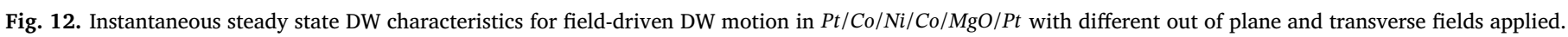
Only the collective coordinate models with highest accuracy in predicting the DW velocity are shown. 


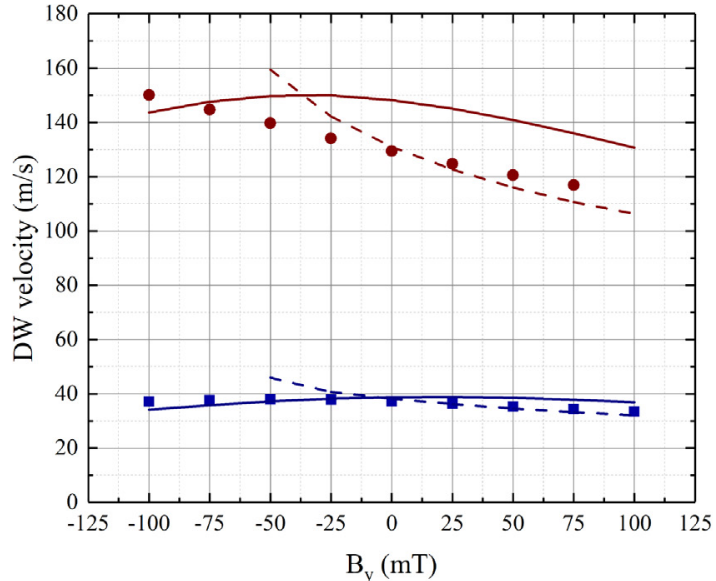

(a) DW velocity.

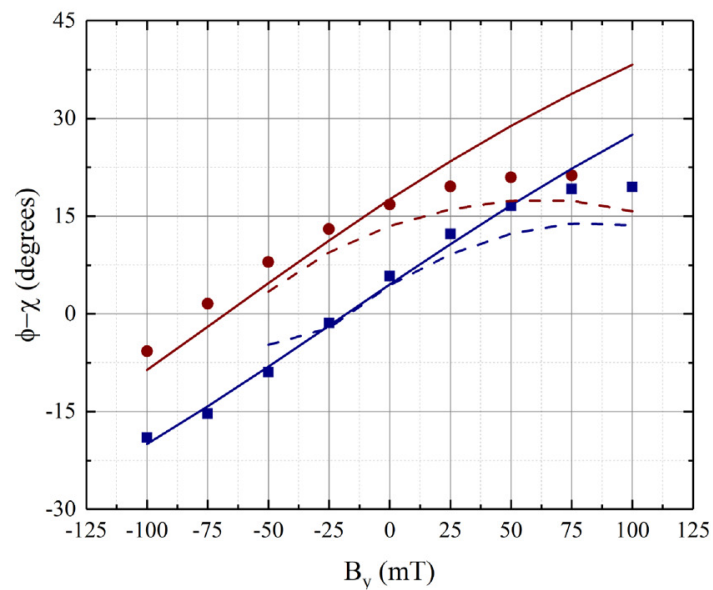

(c) $\phi-\chi$

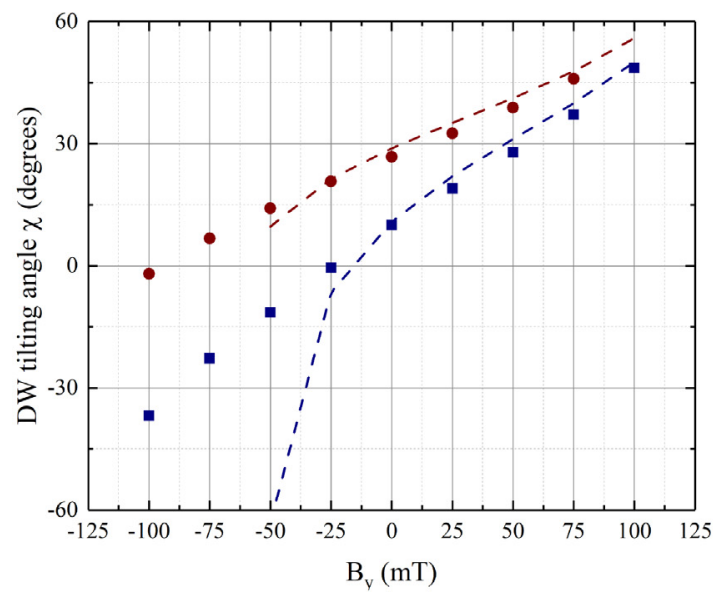

(e) DW tilting angle $(\chi)$.

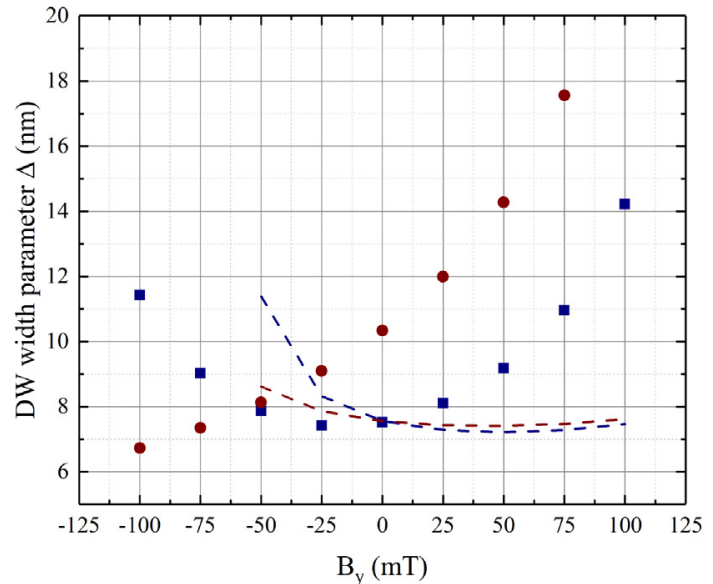

(b) DW width parameter $(\Delta)$.

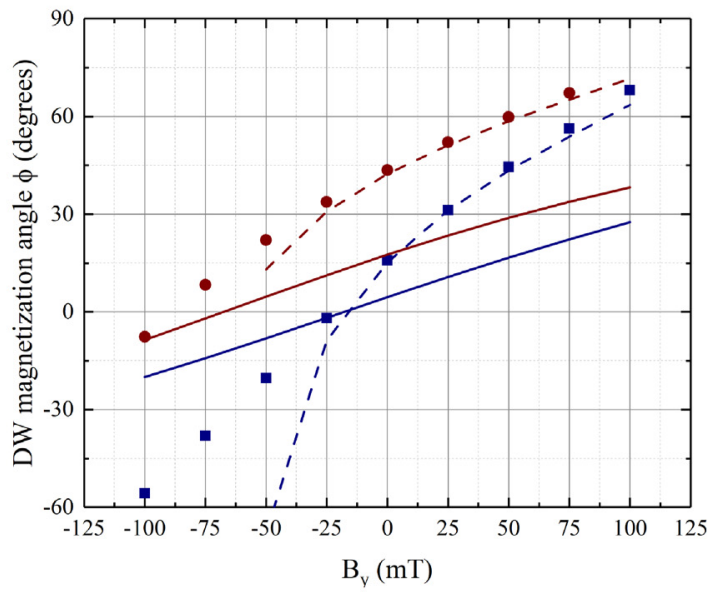

(d) DW magnetization angle $(\phi)$.
- $\mathrm{J}=0.1 \mathrm{TA} / \mathrm{m}^{2}, \mu \mathrm{mag}$

- $\mathrm{J}=0.4 \mathrm{TA} / \mathrm{m}^{2}, \mu \mathrm{mag}$

- - $\mathrm{J}=0.1 \mathrm{TA} / \mathrm{m}^{2}, \mathrm{q}-\phi-\chi-\Delta \operatorname{model} 3$

- - $\mathrm{J}=0.4 \mathrm{TA} / \mathrm{m}^{2}, \mathrm{q}-\phi-\chi-\Delta$ model 3

$-\mathrm{J}=0.1 \mathrm{TA} / \mathrm{m}^{2}, \mathrm{q}-\phi$ model 3

$-\mathrm{J}=0.4 \mathrm{TA} / \mathrm{m}^{2}, \mathrm{q}-\phi$ model 3

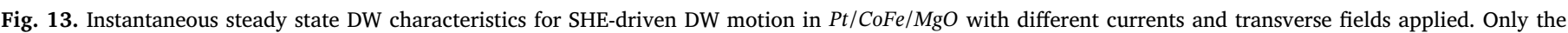
collective coordinate models with highest accuracy in predicting the DW velocity are shown. 


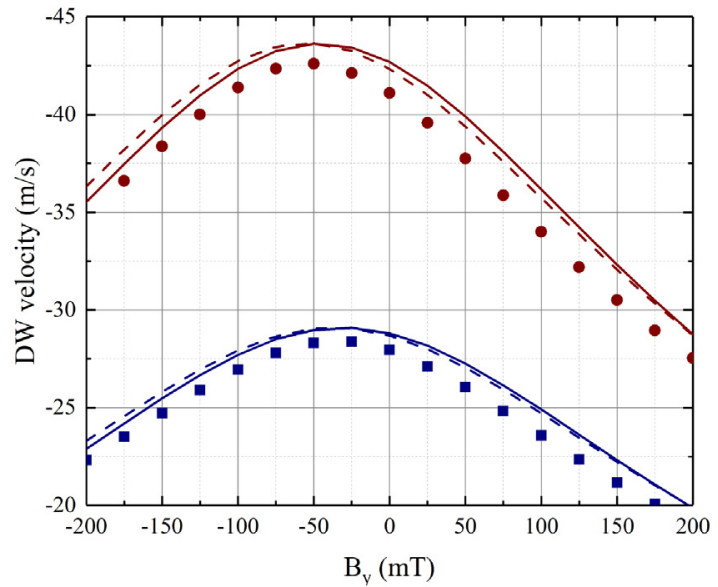

(a) DW velocity.

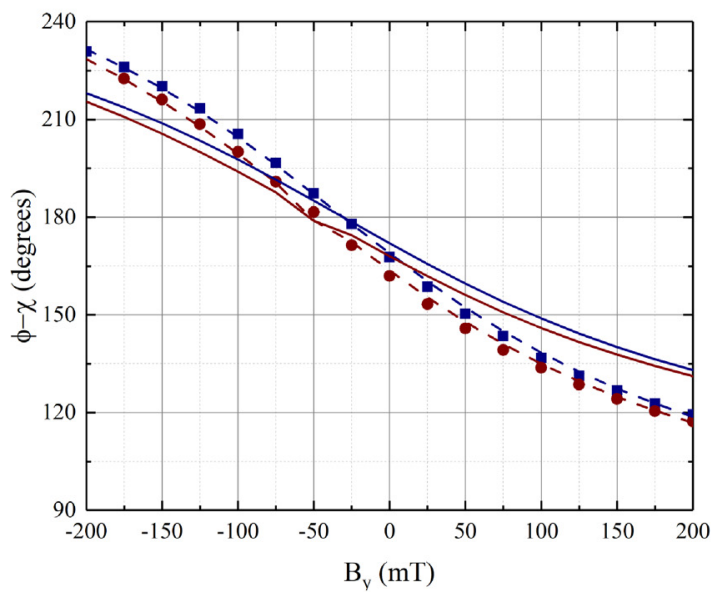

(c) $\phi-\chi$

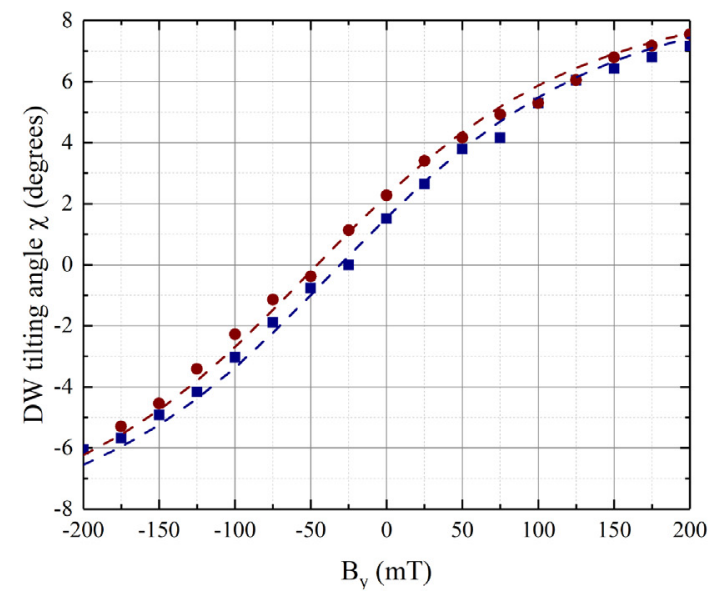

(e) DW tilting angle $(\chi)$.

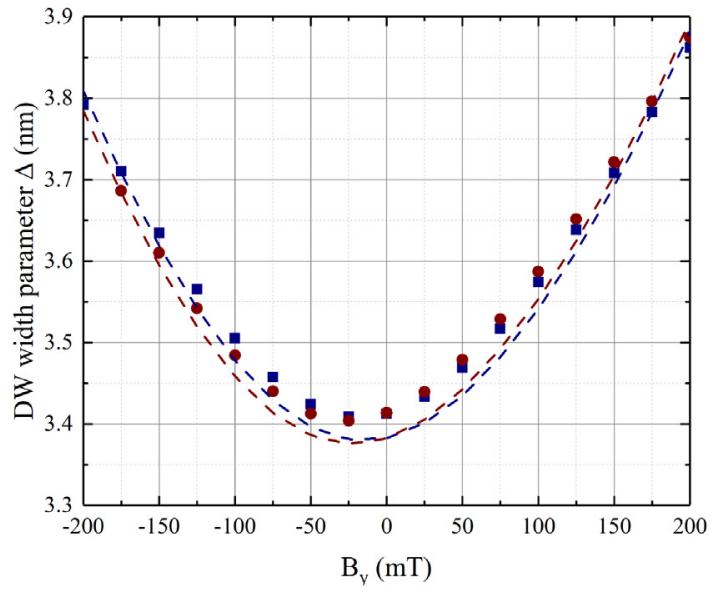

(b) DW width parameter $(\Delta)$.

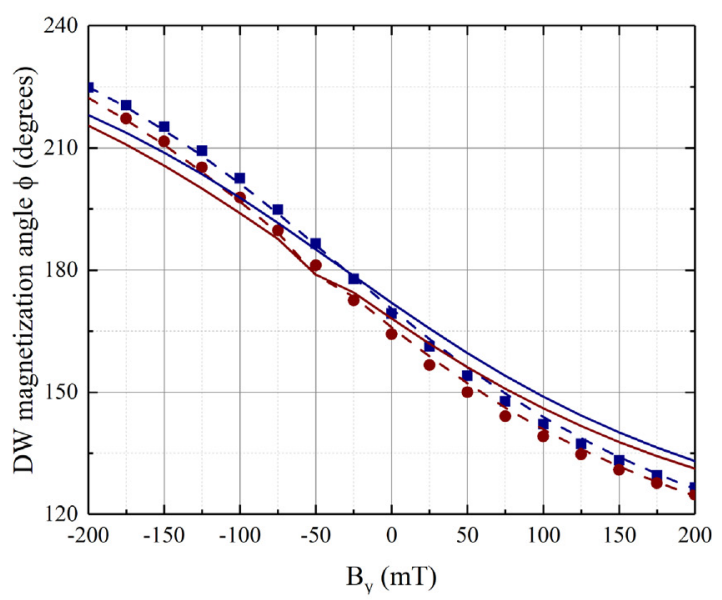

(d) DW magnetization angle $(\phi)$.
- $\mathbf{J}=0.1 \mathrm{TA} / \mathrm{m}^{2}, \mu \mathrm{mag}$

- $\mathrm{J}=0.15 \mathrm{TA} / \mathrm{m}^{2}, \mu \mathrm{mag}$

- - $\mathrm{J}=0.1 \mathrm{TA} / \mathrm{m}^{2}, \mathrm{q}-\phi-\chi-\Delta$ model 1

$---\mathrm{J}=0.15 \mathrm{TA} / \mathrm{m}^{2}, \mu \mathrm{mag}, \mathrm{q}-\phi-\chi-\Delta$ model 1

$-\mathrm{J}=0.1 \mathrm{TA} / \mathrm{m}^{2}, \mathrm{q}-\phi$ model 1

$-\mathrm{J}=0.15 \mathrm{TA} / \mathrm{m}^{2}, \mathrm{q}-\phi$ model 1

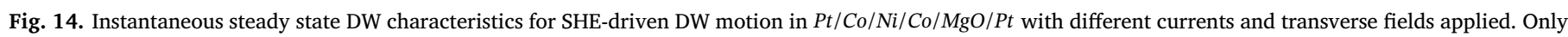
the collective coordinate models with highest accuracy in predicting the DW velocity are shown. 


$$
\begin{aligned}
& \left(I_{2}+\alpha^{2} \frac{I_{1} I_{4}}{I_{2}}\right) I_{1} \frac{\dot{q}}{\mu_{0} \gamma p_{w} \Delta} \\
& \quad= \begin{cases}\alpha\left(I_{4} \frac{H_{z}}{\cos \chi}-\frac{I_{3} I_{4}}{I_{2}} H_{S L}\left[\tan \chi u_{S O T, x}-u_{S O T, y}\right]\right) \\
+\left(\alpha \beta \frac{I_{1} I_{4}}{I_{2}}+I_{5}\right) \frac{u}{p_{w} \Delta}+I_{6}\left[H_{x} \tan \chi-H_{y}\right] & \text { for } \phi-\chi \equiv 0 \\
\alpha\left(I_{4} \frac{H_{z}}{\cos \chi}+\frac{I_{3} I_{4}}{I_{2}} H_{S L}\left[\tan \chi u_{S O T, x}-u_{S O T, y}\right]\right) \\
+\left(\alpha \beta \frac{I_{1} I_{4}}{I_{2}}+I_{5}\right) \frac{u}{p_{w} \Delta}-I_{6}\left[H_{x} \tan \chi-H_{y}\right] & \text { for } \phi-\chi \equiv 180^{\circ}\end{cases}
\end{aligned}
$$

Under the application of transverse fields, the equations become slightly more simplified, as at the same time $\chi=0$, which yields:

$$
\begin{aligned}
& \left(I_{2}+\alpha^{2} \frac{I_{1} I_{4}}{I_{2}}\right) \frac{I_{1}}{\mu_{0} \gamma p_{w} \Delta} \dot{q} \\
& \quad=\left\{\begin{array}{l}
\alpha\left(I_{4} H_{z}+\frac{I_{3} I_{4}}{I_{2}} H_{S L} u_{S O T, y}\right) \\
+\left(\alpha \beta \frac{I_{1} I_{4}}{I_{2}}+I_{5}\right) \frac{u}{p_{w} \Delta}-I_{6} H_{y} \quad \text { if } \phi-\chi \equiv 0(D<0) \\
\alpha\left(I_{4} H_{z}-\frac{I_{3} I_{4}}{I_{2}} H_{S L} u_{S O T, y}\right) \\
+\left(\alpha \beta \frac{I_{1} I_{4}}{I_{2}}+I_{5}\right) \frac{u}{p_{w} \Delta}+I_{6} H_{y} \text { if } \phi-\chi \equiv 180^{\circ}(D>0)
\end{array}\right.
\end{aligned}
$$

Eqs. (9) and (10) are thought-provoking, as they connect measured properties of the DW (DW velocity and tilting) to parameters arising from material properties such as the DW width parameter. These equations can be used to measure specific properties of the DW. Recent experiments have shown the visualization of DW tilting under experimental conditions [51]. In an experimental setting, first a transverse field should be identified at which the DW tilting is negligible; in this condition one may assume based on our results that $\phi \sim \chi \sim 0$. Using field-driven DW motion measurements, Eq. (10) may be used to measure the DW width parameter, which can in turn help estimate the exchange constant through $\Delta=\sqrt{\frac{A}{K}}$. In a current-driven case, the same equation could be used to estimate the SHE angle.

In current-driven DW motion, we identified a longitudinal field for which DW velocity is zero. In $\mathrm{Pt} / \mathrm{CoFe} / \mathrm{MgO}$, this field was about $B_{x} \sim-200 \mathrm{mT}$, while in $\mathrm{Pt} / \mathrm{CoFe} / \mathrm{MgO}$ it was $B_{x} \sim 200 \mathrm{mT}$. We had shown in our previous work that this field is related to the DMI strength [17]. Under these conditions, the DW velocity equation simplifies to:

$$
\begin{aligned}
& \alpha \frac{I_{3} I_{4}}{I_{2}} H_{S L}\left[\cos \phi u_{S O T, y}-\sin \phi u_{S O T, x}\right]+\left(\alpha \beta \frac{I_{1} I_{4}}{I_{2}}+I_{5}\right) \frac{u}{p_{w} \Delta} \cos \chi \\
& =\frac{1}{2} I_{4} M_{s}\left(N_{x}-N_{y}\right) \sin 2(\phi-\chi)+I_{3} \frac{D}{\mu_{0} M_{s} p_{w} \Delta} \sin (\phi-\chi) \\
& \quad-I_{6}\left[H_{x} \sin \phi-H_{y} \cos \phi\right]
\end{aligned}
$$

Looking at Figs. 9 and 10, we also observe that at this in-plane field $\phi$ and $\chi$ seem to be independent of the drive interaction, and $\phi \sim 90^{\circ}$. Using this assumption, we have:

$$
\begin{aligned}
-\alpha \frac{I_{3} I_{4}}{I_{2}} H_{S L} u_{S O T, x}+\left(\alpha \beta \frac{I_{1} I_{4}}{I_{2}}\right. & \left.+I_{5}\right) \frac{u}{p_{w} \Delta} \cos \chi=\frac{1}{2} I_{4} M_{s}\left(N_{x}-N_{y}\right) \sin 2 \chi \\
& +I_{3} \frac{D}{\mu_{0} M_{s} p_{w} \Delta} \cos \chi-I_{6} H_{x}
\end{aligned}
$$

Eq. (12) could be used to measure DMI under conditions which the DW is stationary under applied currents. Plugging observations from the micromagnetic simulations into a two coordinate form of model 3 (and assuming $J=0.1 \mathrm{TA} / \mathrm{m}^{2}$ ), we predict a DMI strength of $D=-1.1 \mathrm{~mJ} / \mathrm{m}^{2}$ for $\mathrm{Pt} / \mathrm{CoFe} / \mathrm{MgO}$ and $D=0.57 \mathrm{~mJ} / \mathrm{m}^{2}$ for $\mathrm{Pt} / \mathrm{Co} / \mathrm{Ni} / \mathrm{Co} / \mathrm{MgO} / \mathrm{Pt}$, which are very close to the values used in the micromagnetic simulations.

In the $\mathrm{Pt} / \mathrm{Co} / \mathrm{Ni} / \mathrm{Co} / \mathrm{MgO} / \mathrm{Pt}$ sample, in field-driven DW motion $\left(B_{z}=30 \mathrm{mT}\right)$, we observed initiation of Walker Breakdown at $B_{x}=50 \mathrm{mT}$ and cessation of this behavior at $B_{x}=325 \mathrm{mT}$. This scenario could be studied using our CCMs. Assuming small tilting for the DW (which is valid in this case), we can simplify the steady state equation as:

$$
\begin{aligned}
\cos \phi= & \frac{I_{2} H_{z}+I_{3} H_{S L} \cos \phi}{\alpha \frac{I_{4}^{2}}{I_{2}} M_{S}\left(N_{y}-N_{x}\right) \sin \phi}+\alpha \frac{\frac{I_{3} I_{4}}{I_{2}} \frac{D}{\mu_{0} M_{s} p_{w} \Delta}+\frac{I_{4} I_{6}}{I_{2}}\left(H_{y} \cot \phi-H_{x}\right)}{\alpha \frac{I_{4}^{2}}{I_{2}} M_{s}\left(N_{y}-N_{x}\right)} \\
= & \frac{1}{2}\left[\left(\frac{I_{2}}{I_{4}}\right)^{2} \frac{H_{z}}{H_{w} \sin \phi}+\frac{I_{2} I_{3}}{I_{4}^{2}} \frac{H_{S L}}{H_{w}} \cot \phi+\alpha \frac{I_{3}}{I_{4}} \frac{H_{D M I}}{H_{w}}-\alpha \frac{I_{6}}{I_{4}} \frac{H_{x}}{H_{w}}\right. \\
& \left.+\alpha \frac{I_{6}}{I_{4}} \frac{H_{y}}{H_{w}} \cot \phi\right]
\end{aligned}
$$

where $H_{w}$ is the conventional Walker Breakdown field [48], and $H_{D M I}$ is the DMI field. Walker Breakdown happens when the right side of the equation above is larger than 1 or smaller than -1 . While in systems without DMI, only the drive interaction and the demagnetizing field played a role in this solution, in a system with DMI and in-plane fields additional terms are introduced; the relevant strength of these terms compared to each other determined whether Walker breakdown will take place or not. Note that magnetocrystalline anisotropy plays a role in this through the $I_{i}$ values, as these values depend on $\theta_{c}$ which in turn depends on $K_{u}$.

Finally, we also observed in-plane fields that led to switching of the system through the elastic extension of the DW. The threshold for this switching field seems to relate to the canting angle reaching $\theta_{c}=45^{\circ}$ at which point the DW does not maintain a rigid structure as the domains try to fully align with the external magnetic field. This leads to $H_{x, s}=\frac{\sqrt{2}}{2}\left[\frac{2 K_{u}}{\mu_{0} M_{s}}+M_{s}\left(N_{x}-N_{z}\right)\right]$ for the longitudinal switching field and $H_{y, s}=\frac{\sqrt{2}}{2}\left[\frac{2 K_{u}}{\mu_{0} M_{s}}+M_{s}\left(N_{y}-N_{z}\right)\right]$ for transverse fields. These equations are expected to over-predict the switching field, as they do not take into account edge effects in the system and the effect of the drive interaction.

For the $\mathrm{Pt} / \mathrm{CoFe} / \mathrm{MgO}$ system, $B_{x, s} \approx \pm 354 \mathrm{mT}$ which is only 25-50 $\mathrm{mT}$ higher than the field at which the system switched in micromagnetic simulations for positive longitudinal fields. For negative longitudinal fields, switching could not be observed due to the nucleation of a new DW. The transverse switching field was found to be $B_{y, s} \approx \pm 359 \mathrm{mT}$ depending on the width of the system; however, we observed elongations in the DW prior to reaching such high fields, albeit these elongations were seen in conjunction with translational motion of the DW. The nature of these elongations and their modeling is beyond the scope of this work, as our CCMs assume the DW is a rigid object. We did not observe any of these effects for the $\mathrm{Pt} / \mathrm{Co} / \mathrm{Ni} / \mathrm{Co} / \mathrm{MgO} / \mathrm{Pt}$ cases; we verified that the switching field for this sample under both longitudinal and transverse fields is about $\pm 1.48 T$, well above the in-plane field values studied.

\subsection{Selecting the Right CCM}

The results of the micromagnetic simulations presented in Figs. 7-14 highlighted the importance of using the right CCM when studying different systems.

First, we found that canting in the domains should be included in the model only if canting in the domains is larger than about $10^{\circ}$; otherwise its inclusion does not add value to the models and can overcomplicate the study. Hence, we recommend using the canted models only when $\theta_{c}>10^{\circ}$ is expected.

We also found that model set 3 is more applicable without the $\Delta$ degree of freedom, as it does not predict this parameter correctly and seems to be of a more rigid nature than the Bloch profile. This is understandable from a modeling perspective, as $\Delta$ in a way determines the transition from DW to domain, and canting impacts the domain's structure.

Finally, most of our models are adept at predicting the right tilting 
and magnetization angles at the critical in-plane fields identified. As such, use of these critical points when trying to identify material properties from the collective coordinate models is recommended.

Overall, it seems that when studying the velocity of the domain wall under longitudinal fields, use of the $q-\phi$ form of model 3 is sufficient, while under transverse fields or other cases where predicting the DW tilting is of interest, the $q-\phi-\chi$ form of model 3 or the $q-\phi-\chi-\Delta$ form of model 2 should be used.

\section{Conclusion}

In this paper, we studied DW motion in PMA materials with DMI under the application of in-plane fields. We showed how the application of moderate in-plane fields could change the dynamics of domain walls by adjusting the internal structure of the DW (magnetization and DW width) along with the tilting of the DW.

A new extended collective coordinate model was introduced taking into account the effect of canting in the domains and was compared to other models present in the literature. Canting was found to play an important role in some systems (depending on their anisotropy), and is a parameter that needs to be factored in any calculations involving collective coordinate models under in-plane fields. We observed that a two coordinate $q-\phi$ model including the effect of canting through a canted ansatz would suffice for studying the DW velocity, while a more complex $q-\phi-\chi-\Delta$ model using the Bloch profile should be used when studying the DW tilting angle is of interest.

Several critical in-plane fields were identified, under which the DW behaves in a predictable way. These include a case where the DW does not tilt, a case where the DW velocity becomes zero in current-driven DW motion, and cases where the intenral structure of the DW is effectively Néel. We derived analytical descriptions for these cases which connect material properties to measurable DW features and could be used in estimating specific features of the materials under study.

\section{Acknowledgements}

This study was conducted as part of the Marie Currie ITN WALL project, which has received funding from the European Union's Seventh Framework Programme for research, technological development and demonstration under grant agreement No. 608031. The work by E. M. was also supported by projects MAT2014- 52477-C5-4-P, MAT201787072-C4-1-P, and MAT2017-90771-REDT from the Spanish government, and projects SA282U14 and SA090U16 from the Junta de Castilla y Leon.

\section{References}

[1] D.A. Allwood, G. Xiong, C.C. Faulkner, D. Atkinson, D. Petit, R.P. Cowburn, Magnetic domain-wall logic, Science 309 (5741) (2005) 1688-1692, https://doi. org/10.1126/science.1108813.

[2] L. Thomas, M. Hayashi, R. Moriya, C. Rettner, S. Parkin, Topological repulsion between domain walls in magnetic nanowires leading to the formation of bound states, Nat. Commun. 3 (810). doi:https://doi.org/10.1038/ncomms1808.

[3] C. Chappert, A. Fert, F.N. Van Dau, The emergence of spin electronics in data storage, Nat. Mater. 6 (11) (2007) 813-823, https://doi.org/10.1038/nmat2024.

[4] S.P. Parkin, M. Hayashi, L. Thomas, Magnetic domain-wall racetrack memory, Science 320 (5873) (2008) 190-194, https://doi.org/10.1126/science.1145799.

[5] S. Parkin, S.H. Yang, Memory on the racetrack, Nat. Nanotechnol. 10 (2015) 195-198, https://doi.org/10.1038/nnano.2015.41.

[6] K.W. Moon, D.H. Kim, S.C. Yoo, S.G. Je, B.S. Chun, W. Kim, B.C. Min, C. Hwang, S. B. Choe, Magnetic bubblecade memory based on chiral domain walls, Sci. Rep. 5 (9166). doi:https://doi.org/10.1038/srep09166.

[7] J. Katine, E.E. Fullerton, Device implications of spin-transfer torques, J. Magn. Magn. Mater. 320 (2008) 1217-1226, https://doi.org/10.1016/j.jmmm.2007.12. 013.

[8] J.C. Slonczewski, Dynamics of magnetic domains, AIP Conf. Proc. 5 (1972) 170-174, https://doi.org/10.1063/1.3699416.

[9] A.A. Thiele, Steady-state motion of magnetic domains, Phys. Rev. Lett. 30 (1973) 230-233, https://doi.org/10.1103/PhysRevLett.30.230.

[10] A.A. Thiele, F.B. Hagedorn, G.P. Vella-Coleiro, Dynamic spin configuration for hard magnetic bubbles in translational motion, Phys. Rev. B 8 (1973) 241-245, https://
doi.org/10.1103/PhysRevB.8.241.

[11] A. Thiaville, Y. Nakatani, J. Miltat, N. Vernier, Domain wall motion by spin-polarized current: a micromagnetic study, J. Appl. Phys. 95 (2004) 7049-7051, https://doi.org/10.1063/1.1667804.

[12] A. Thiaville, Y. Nakatani, J. Miltat, Y. Suzuki, Micromagnetic understanding of current-driven domain wall motion in patterned nanowires, Europhys. Lett. 69 (2005) 990-996, https://doi.org/10.1209/epl/i2004-10452-6.

[13] A. Thiaville, Y. Nakatani, Spin Dynamics in Confined Magnetic Structures III, Vol Volume 101/2006 of Topics in Applied Physics, Springer, 2006, Ch. Domain-Wall Dynamics in Nanowires and Nanostrips, pp. 161-205. doi:https://doi.org/10.1007/ 10938171_5.

[14] O. Boulle, S. Rohart, L.D. Buda-Prejbeanu, E. Jué, I.M. Miron, S. Pizzini, J. Vogel, G. Gaudin, A. Thiaville, Domain wall tilting in the presence of the DzyaloshinskiiMoriya interaction in out-of-plane magnetized magnetic nanotracks, Phys. Rev. Lett. 111 (217203). doi:https://doi.org/10.1103/PhysRevLett.111.217203.

[15] J. Vandermeulen, S.A. Nasseri, B.V. de Wiele, G. Durin, B.V. Waeyenberge, L. Dupre, The effect of Dzyaloshinskii-Moriya interaction on field-driven domain wall dynamics analysed by a semi-analytical approach, J. Phys. D: Appl. Phys. 49 (465003). doi:https://doi.org/10.1088/0022-3727/49/46/465003.

[16] J. Vandermeulen, S.A. Nasseri, B.V. de Wiele, G. Durin, B.V. Waeyenberge, L. Dupre, Comparison between collective coordinate models for domain wall motion in PMA nanostrips in the presence of the Dzyaloshinskii-Moriya interaction, J. Magn. Magn. Mater. 449 (2018) 337-352, https://doi.org/10.1016/j.jmmm.2017. 10.008 .

[17] S.A. Nasseri, S. Moretti, E. Martinez, C. Serpico, G. Durin, Collective coordinate models of domain wall motion in perpendicularly magnetized systems under the spin Hall effect and longitudinal fields, J. Magn. Magn. Mater. 426 (2017) 195-201, https://doi.org/10.1016/j.jmmm.2016.11.081.

[18] A.K. Nguyen, H.J. Skadsem, A. Brataas, Giant current-driven domain wall mobility in (Ga,Mn)As, Phys. Rev. Lett. 98 (146602). doi:https://doi.org/10.1103/ PhysRevLett.98.146602.

[19] A. Manchon, S. Zhang, Theory of nonequilibrium intrinsic spin torque in a single nanomagnet, Phys. Rev. B 78 (212405). doi:https://doi.org/10.1103/physrevb.78. 212405.

[20] A. Matos-Abiague, R.L. Rodriguez-Suarez, Spin-orbit coupling mediated spin torque in a single ferromagnetic layer, Phys. Rev. B 80 (094424). doi:https://doi.org/10. 1103/PhysRevB.80.094424.

[21] A. Chernyshov, M. Overby, X. Liu, J.K. Furdyna, Y. Lyanda-Geller, L.P. Rokhinson, Evidence for reversible control of magnetization in a ferromagnetic material by means of spin-orbit magnetic field, Nat. Phys. 5 (2009) 656-659, https://doi.org/ 10.1038/nphys1362.

[22] P.P.J. Haazen, E. Muré, J.H. Franken, R. Lavrijsen, H.J.M. Swagten, B. Koopmans, Domain wall depinning governed by the spin hall effect, Nat. Mater. 12 (2013) 299-303, https://doi.org/10.1038/nmat3553.

[23] E. Martinez, S. Emori, G.S.D. Beach, Current-driven domain wall motion along high perpendicular anisotropy multilayers: the role of the rashba field, the spin Hall effect, and the Dzyaloshinskii-Moriya interaction, Appl. Phys. Lett. 103. doi:https:// doi.org/10.1063/1.4818723.

[24] E. Martinez, S. Emori, N. Perez, L. Torres, G.S.D. Beach, Current-driven dynamics of Dzyaloshinskii domain walls in the presence of in-plane fields: full micromagnetic and one-dimensional analysis, J. Appl. Phys. 115 (213909). doi:https://doi.org/10. 1063/1.4881778.

[25] O. Boulle, L.D. Buda-Prejbeanu, M. Miron, G. Gaudin, Current induced domain wall dynamics in the presence of a transverse magnetic field in out-of-plane magnetized materials, J. Appl. Phys. 112 (053901). doi:https://doi.org/10.1063/1.4747907.

[26] O. Boulle, L.D. Buda-Prejbeanu, E. Jué, I.M. Miron, G. Gaudin, Current induced domain wall dynamics in the presence of spin orbit torques, J. Appl. Phys. 115 (17D502). doi:https://doi.org/10.1063/1.4860946.

[27] S. Emori, U. Bauer, S.-M. Ahn, E. Martinez, G.S.D. Beach, Current-driven dynamics of chiral ferromagnetic domain walls, Nat. Mater. 12 (2013) 611-616, https://doi. org/10.1038/nmat3675.

[28] S. Emori, E. Martinez, K.-J. Lee, H.-W. Lee, U. Bauer, S.-M. Ahn, P. Agrawal, D.C. Bono, G.S.D. Beach, Spin Hall torque magnetometry of Dzyaloshinskii domain walls, Phys. Rev. B 90 (184427). doi:https://doi.org/10.1103/PhysRevB.90. 184427.

[29] S.A. Nasseri, B. Sarma, G. Durin, C. Serpico, Analytical modelling of magnetic DW motion, Phys. Procedia 75 (2015) 974-985, https://doi.org/10.1016/j.phpro.2015. 12.133 Proceedings of the 20th International Conference on Magnetism - ICM 2015

[30] S.-G. Je, D.-H. Kim, S.-C. Yoo, B.-C. Min, K.-J. Lee, S.-B. Choe, Asymmetric magnetic domain-wall motion by the Dzyaloshinskii-Moriya interaction, Phys. Rev. B 88 (214401). doi:https://doi.org/10.1103/PhysRevB.88.214401.

[31] A. Hrabec, N.A. Porter, A. Wells, M.J. Benitez, G. Burnell, S. McVitie, D. McGrouther, T.A. Moore, C.H. Marrows, Measuring and tailoring the Dzyaloshinskii-Moriya interaction in perpendicularly magnetized thin films, Phys. Rev. B 90 (020402). doi:https://doi.org/10.1103/PhysRevB.90.020402.

[32] Y. Yoshimura, K.-J. Kim, T. Taniguchi, T. Tono, K. Ueda, R. Hiramatsu, T. Moriyama, K. Yamada, Y. Nakatani, T. Ono, Soliton-like magnetic domain wall motion induced by the interfacial dzyaloshinskii-moriya interaction, Nat. Phys. 12 (2016) 157-161, https://doi.org/10.1038/nphys3535.

[33] A. Vansteenkiste, J. Leliaert, M. Dvornik, M. Helsen, F. Garcia-Sanchez, B. Van Waeyenberge, The design and verification of MuMax3, AIP Adv. 4 (107133). doi:https://doi.org/10.1063/1.4899186.

[34] A.N. Bogdanov, D.A. Yablonskii, Thermodynamically stable vortices in magnetically ordered crystals. the mixed state of magnets, Sov. Phys. JETP 68 (1989).

[35] A.N. Bogdanov, U.K. Rößler, Chiral symmetry breaking in magnetic thin films and multilayers, Phys. Rev. Lett. 87. doi:https://doi.org/10.1103/physrevlett.87. 
037203.

[36] A. Thiaville, S. Rohart, E. Jué, V. Cros, A. Fert, Dynamics of dzyaloshinskii domain walls in ultrathin magnetic films, Europhys. Lett. 100 (57002). doi:https://doi.org/ 10.1209/0295-5075/100/57002.

[37] P.M. Haney, H.-W. Lee, K.-J. Lee, A. Manchon, M.D. Stiles, Current-induced torques and interfacial spin-orbit coupling, Phys. Rev. B 88 (2013) 214417, https://doi.org/ 10.1103/PhysRevB.88.214417.

[38] K. Garello, I.M. Miron, C.O. Avci, F. Freimuth, Y. Mokrousov, S. Bluegel, S. Auffret, O. Boulle, G. Gaudin, P. Gambardella, Symmetry and magnitude of spin-orbit torques in ferromagnetic heterostructures, Nat. Nanotechnol. 8 (2013) 587-593, https://doi.org/10.1038/NNANO.2013.145.

[39] A.V. Khvalkovskiy, V. Cros, D. Apalkov, V. Nikitin, M. Krounbi, K.A. Zvezdin, A. Anane, J. Grollier, A. Fert, Matching domain-wall configuration and spin-orbit torques for efficient domain-wall motion, Phys. Rev. B 87 (020402). doi:https://doi. org/10.1103/PhysRevB.87.020402.

[40] S. Zhang, Z. Li, Roles of nonequilibrium conduction electrons on the magnetization dynamics of ferromagnets, Phys. Rev. Lett. 93 (127204). doi:https://doi.org/10. 1103/physrevlett.93.127204.

[41] V.L. Sobolev, S.C. Chen, H.L. Huang, Equations of domain wall motion in ferromagnetic medium with $q>1$, IEEE Trans. Magn. 29 (1993) 2542-2544, https:// doi.org/10.1109/20.280960.

[42] V.L. Sobolev, H.L. Huang, S.C. Chen, Generalized equations for domain wall dynamics, J. Appl. Phys. 75 (1994) 5797-5799, https://doi.org/10.1063/1.355566.

[43] V.L. Sobolev, H.L. Huang, S.C. Chen, Domain wall dynamics in the presence of an external magnetic field normal to the anisotropy axis, J. Magn. Magn. Mater. 147 (1995) 284-298, https://doi.org/10.1016/0304-8853(95)00065-8.
[44] V.L. Sobolev, H.L. Huang, New equations for the domain wall dynamics, J. Magn. Magn. Mater. 140-144 (1995) 1849-1850, https://doi.org/10.1016/0304-8853(94) 01602-x.

[45] D.C. Cronemeyer, Demagnetization factors for general ellipsoids, J. Appl. Phys. 70 (1991) 2911-2914, https://doi.org/10.1063/1.349315.

[46] M. Vanatka, J.-C. Rojas-Sánchez, J. Vogel, M. Bonfim, M. Belmeguenai, Y. Roussigné, A. Stashkevich, A. Thiaville, S. Pizzini, Velocity asymmetry of Dzyaloshinskii domain walls in the creep and flow regimes, J. Phys.: Condens. Matter 27 (326002). doi:https://doi.org/10.1088/0953-8984/27/32/326002.

[47] T. Tono, T. Taniguchi, K.-J. Kim, T. Moriyama, A. Tsukamoto, T. Ono, Chiral magnetic domain wall in ferrimagnetic GdFeCo wires, Appl. Phys. Express 8 (073001). doi:https://doi.org/10.7567/apex.8.073001.

[48] N.L. Schryer, L.R. Walker, The motion of $180^{\circ}$ domain walls in uniform dc magnetic fields, J. Appl. Phys. 45 (1974) 5406-5421, https://doi.org/10.1063/1.1663252.

[49] K.S. Ryu, L. Thomas, S.H. Yang, S. Parkin, Chiral spin torque at magnetic domain walls, Nat. Nanotechnol. 8 (2013) 527-533, https://doi.org/10.1038/nnano.2013. walls, 102.

[50] K.-S. Ryu, S.-H. Yang, L. Thomas, S.S.P. Parkin, Chiral spin torque arising from proximity-induced magnetization, Nat. Commun. 5 (3910). doi:https://doi.org/10. 1038/ncomms4910.

[51] J. Yun, D. Li, B. Cui, X. Guo, K. Wu, X. Zhang, Y. Wang, J. Mao, Y. Zuo, L. Xi, Current induced domain wall motion and tilting in ptcota structures with perpendicular magnetic anisotropy in the presence of the Dyzaloshinskii-Moriya interaction, J. Phys. D: Appl. Phys. 51 (155001). doi:https://doi.org/10.1088/1361-6463/ aab419. 